\title{
VASCULAR FLORA IN CEMETERIES OF THE ROZTOCZE REGION AND SURROUNDING AREAS (SOUTH-EAST POLAND)
}

\author{
Aneta Czarna, Renata Nowińska \\ Department of Botany, Poznań University of Life Sciences, Wojska Polskiego 71c, 60-625 Poznań, Poland, \\ e-mail: czarna@up.poznan.pl
}

Received: 05.09.2010

\begin{abstract}
The paper presents a systematic list of vascular plant species recorded at 78 cemeteries in the Roztocze region and surrounding areas. 543 species belonging to 75 families were recorded. Of these, 99 foreign and 43 native species were cultivated. 41 species introduced by humans to cemeteries can be regarded as the so-called established cemetery species. These species, once planted on graves, continue to grow or even spread after people stopped cultivating them.
\end{abstract}

Key words: vascular flora, cemetery, the Roztocze region, Poland

\section{INTRODUCTION}

Cemeteries constitute a material representation of history, traditions and beliefs of the local population. The diversity and exceptional character of cemeteries are determined first of all by architectural aspects, e.g. their location, shape, spatial arrangement and the type of gravestones. The unique character of a necropolis is also connected with the so-called cemetery green, which is affected not only by the original and planned arrangement, but also the inventiveness and aesthetic sense of individuals taking care of individual gravestones and the whole cemeteries. Cemeteries are important localities of plants in rural and urban floras (S u dnik-W ó j cik ow ska, 1987; B randes and B randes, 1996; M a ciej czak, 2008).

Cemeteries in the Roztocze region are the most beautiful not only on the national scale, but also throughout Europe; however, they are relatively unknown (Kolbuszewski, 1996). They are mostly old cemeteries, located amid fields and forests. A characteristic feature of the Roztocze cemeteries (particularly those from the Eastern Roztocze region) is the occur- rence of folk art crosses and gravestones made from Tertiary sandstone (the so-called gravestone carving from the Bruśno stoneworking centre). They may be found in numerous cemeteries of different denominations: Greek Catholic and Roman Catholic (W ł a d and $\mathrm{W}$ iśn i ew s k i, 2004). Many old cemeteries in that region of Poland have still retained rich old stands. They are also localities of plants grown in former times as well as of rare and interesting species.

This paper presents results of studies on vascular flora of 78 cemeteries from the Roztocze region and surrounding areas (Płaskowyż Tarnogrodzki, Równina Bełska) (Fig. 1, Table 1). 36 of the studied cemeteries are in active use, while 42 are currently closed and regarded as monuments. A smaller part of these temporary cemeteries (for example the ones located in Lubaczów and its vicinity, Podlesie) were used to bury German Evangelical settlers; the survivors among them left Poland during WW II. A greater part of these temporary cemeteries (for example the ones located in Łówcza, Gorajec, Chotylub, Huta Różaniecka) were used to bury the Ukrainian Greek Catholic population; its members were displaced from this region during the 1944-1947 period.

The aim of the study was to determine a list of vascular plant species in the analysed cemeteries, to conduct the analysis of the collected material and to establish a list of permanent cemetery species.

\section{MATERIAL AND METHODS}

Floristic inventories were conducted in the growing season of 2007. A total of 5004 floristic data were collected.

It included a list of families and species, with plant families presented using a system according to 
the key proposed by R u tk ow s k i (1998), while the species were presented alphabetically. Nomenclature of species was adopted after Mirek et al. (2002). Square brackets contain information on their geographic-historical status, life form and socio-ecological group. Parentheses with Roman numerals contain the degree of cover in a 5-point scale, provided that the degree of cover was at least II. Arabic numerals outside the parentheses denote the numbers assigned to the cemeteries in which a given species was recorded (the list of cemeteries is presented in Fig. 1, Table 1).

Geographic-historical groups were presented according to Chmiel (1993), with slight modifications: Sp - spontaneophytes; Ap - apophytes; Arch archaeophytes; Ken -kenophytes; D - diaphytes.

Life forms sensu Raunkiaer were adopted after Z a r z y cki et al. (2002): M - megaphanerophytes; $\mathrm{N}$ - nanophanerophytes; $\mathrm{Ch}$ - chamaephytes; $\mathrm{H}$ - hemicryptophytes; $\mathrm{G}$ - geophytes; $\mathrm{T}$ - therophytes; Hel - helophytes.

Socio-ecological groups were presented according to Chmiel (1993): 1 - Fagetalia, Prunetalia; 2 - Quercion robori-petraeae, Epilobion, Nardetalia; 3 -Sambuco-Salicion, Alliarion; 4 - Trifolio-Geranietea, Festuco-Brometea; 5 - Dicrano-Pinion, Sedo-Scleranthetea, Corynephoretea; 6 - Alnion, Magnocaricion, Caricetalia fuscae, Sphagnion fusci; 7 - Salicion,
Phragmition, Glycerio-Sparganion, Potamogetonetea, Lemnetea, Utricularietea; 8 - Molinietalia; 9 -Arrenatheretalia; 10 - Plantaginetea; 11 - Thero-Salicornietea, Asteretea tripolium, 12 - Bidentetea, Nanocyperion; 13 - Arction; 14 - Onopordion; 15 - Sisymbrion, Eragrostion; 16 - Polygono-Chenopodietalia; 17 - Aperetalia; 18 - Asplenietea; 19 - native species or naturalized antropophytes of undetermined phytosociological status and ephemerophytes.

A 5-point scale was adopted, indicating the area covered by a given species: I - the species growing on $<5 \%$ of the cemetery area, II $-5-25 \%$, III $-25-50 \%$, IV $-50-75 \%$ ), and V - 75-100 \%, respectively.

Native currently cultivated species are underlined with a double line, while foreign, formerly cultivated species are underlined with a single line.

The following scale was adopted in the determination of the frequency of species: very rare (1-2 localities), rare (3-5 localities), relatively frequent (6-10 localities), frequent (11-25 localities), very frequent (26-50 localities), and common (51-78 localities).

A list was prepared comprising species that were planted on graves and which, after being abandoned, have been growing in good condition until the present time, or even have been spreading. These species are referred to as constant cemetery plants. These species are good phytoindicators of cemetery locations.

Table 1.

A description of the investigated cemeteries in the Roztocze region. Cemeteries are denoted with numbers as in Fig. 1

\begin{tabular}{|c|c|c|c|c|}
\hline No & Locality & Commune & Cemetery area $(\mathrm{m})$ & Usage \\
\hline 1 & Podlesie & Lubaczów & $25 \times 25$ & $\mathrm{C}$ \\
\hline 2 & Basznia Dolna & Lubaczów & $32 \times 65$ & $\mathrm{C}$ \\
\hline 3 & Bszania Dolna & Lubaczów & $85 \times 60$ & A \\
\hline 4 & Karolówka & Lubaczów & $20 \times 35$ & A \\
\hline 5 & Młodów & Lubaczów & $20 \times 30$ & $\mathrm{C}$ \\
\hline 6 & Lubaczów & Lubaczów & $50 \times 100$ & $\mathrm{~A}$ \\
\hline 7 & Lubaczów & Lubaczów & $30 \times 35$ & $\mathrm{C}$ \\
\hline 8 & Lubaczów & Lubaczów & $25 \times 40$ & $\mathrm{C}$ \\
\hline 9 & Dachnów & Cieszanów & $45 \times 55$ & A \\
\hline 10 & Załuże & Lubaczów & $40 \times 40$ & $\mathrm{~A}$ \\
\hline 11 & Cieszanów & Cieszanów & $80 \times 80$ & A \\
\hline 12 & Kowalówka & Cieszanów & $25 \times 120$ & $\mathrm{C}$ \\
\hline 13 & Ruda Różaniecka & Narol & $25 \times 120$ & $\mathrm{~A}$ \\
\hline 14 & Płazów & Narol & $90 \times 190$ & A \\
\hline 15 & Łówcza & Narol & $60 \times 70$ & $\mathrm{C}$ \\
\hline 16 & Gorajec & Cieszanów & $40 \times 100$ & $\mathrm{C}$ \\
\hline 17 & Nowe Sioło & Cieszanów & $35 \times 70$ & A \\
\hline 18 & Tansówka & Horyniec Zdrój & $35 \times 80$ & $\mathrm{C}$ \\
\hline 19 & Podemszczyzna & Horyniec Zdrój & $30 \times 30$ & $\mathrm{C}$ \\
\hline 20 & Podemszczyzna & Horyniec Zdrój & $30 \times 60$ & $\mathrm{C}$ \\
\hline 21 & Nowe Brusno & Horyniec Zdrój & $30 \times 60$ & $\mathrm{~A}$ \\
\hline 22 & Polanka Horyniecka & Horyniec Zdrój & $25 \times 100$ & $\mathrm{C}$ \\
\hline 23 & Stare Brusno & Horyniec Zdrój & $25 \times 75$ & $\mathrm{C}$ \\
\hline 24 & Chotylub & Cieszanów & $30 \times 60$ & $\mathrm{C}$ \\
\hline
\end{tabular}




\begin{tabular}{|c|c|c|c|c|}
\hline 25 & Horyniec Zdrój & Horyniec Zdrój & $50 \times 150$ & $\mathrm{~A}$ \\
\hline 26 & Radruż & Horyniec Zdrój & $20 \times 35$ & $\mathrm{C}$ \\
\hline 27 & Radruż & Horyniec Zdrój & $40 \times 60$ & $\mathrm{C}$ \\
\hline 28 & Moczary & Horyniec Zdrój & $30 \times 50$ & $\mathrm{~A}$ \\
\hline 29 & Werchrata & Horyniec Zdrój & $45 \times 110$ & $\mathrm{~A}$ \\
\hline 30 & Prusie & Horyniec Zdrój & $50 \times 50$ & $\mathrm{~A}$ \\
\hline 31 & Hrebenne & Lubycza Królewska & $60 \times 60$ & $\mathrm{~A}$ \\
\hline 32 & Siedliska & Lubycza Królewska & $40 \times 100$ & A \\
\hline 33 & Kornie & Lubycza Królewska & $35 \times 150$ & $\mathrm{C}$ \\
\hline 34 & Stary Machnów & Lubycza Królewska & $35 \times 40$ & $\mathrm{C}$ \\
\hline 35 & Wierzbica & Lubycza Królewska & $20 \times 50$ & $\mathrm{C}$ \\
\hline 36 & Nowosiółki Przednie & Lubycza Królewska & $20 \times 40$ & $\mathrm{C}$ \\
\hline 37 & Nowosiółki Kardynalskie & Lubycza Królewska & $30 \times 30$ & $\mathrm{C}$ \\
\hline 38 & Chodywańce & Jarczów & $50 \times 150$ & A \\
\hline 39 & Jurów & Jarczów & $20 \times 30$ & $\mathrm{C}$ \\
\hline 40 & Jarczów & Jarczów & $50 \times 60$ & A \\
\hline 41 & Żurawce & Lubycza Królewska & $30 \times 120$ & A \\
\hline 42 & Żurawce & Lubycza Królewska & $60 \times 70$ & $\mathrm{~A}$ \\
\hline 43 & Mosty Małe & Lubycza Królewska & $30 \times 70$ & $\mathrm{~A}$ \\
\hline 44 & Teniatyska & Lubycza Królewska & $50 \times 150$ & $\mathrm{C}$ \\
\hline 45 & Lubycza Królewska & Lubycza Królewska & $80 \times 100$ & $\mathrm{~A}$ \\
\hline 46 & Lubycza Królewska & Lubycza Królewska & $20 \times 30$ & $\mathrm{C}$ \\
\hline 47 & Lubycza Królewska & Lubycza Królewska & $30 \times 40$ & $\mathrm{C}$ \\
\hline 48 & Kniazie & Lubycza Królewska & $30 \times 40$ & $\mathrm{C}$ \\
\hline 49 & Kniazie & Lubycza Królewska & $30 \times 200$ & $\mathrm{C}$ \\
\hline 50 & Chyże & Bełżec & $50 \times 60$ & $\mathrm{~A}$ \\
\hline 51 & Bełżec & Bełżec & $150 \times 200$ & $\mathrm{~A}$ \\
\hline 52 & Bełżec & Bełżec & $20 \times 40$ & $\mathrm{C}$ \\
\hline 53 & Tomaszów Lubelski & Tomaszów Lubelski & $60 \times 150$ & $\mathrm{C}$ \\
\hline 54 & Tomaszów Lubelski & Tomaszów Lubelski & $200 \times 200$ & $\mathrm{~A}$ \\
\hline 55 & Łaszczówka & Tomaszów Lubelski & $100 \times 100$ & A \\
\hline 56 & Wierszczyca & Jarczów & $20 \times 30$ & $\mathrm{C}$ \\
\hline 57 & Szlatyn & Jarczów & $20 \times 30$ & C \\
\hline 58 & Hubinek & Ulhówek & $30 \times 50$ & $\mathrm{~A}$ \\
\hline 59 & Przeorsk & Tomaszów Lubelski & $20 \times 50$ & $\mathrm{C}$ \\
\hline 60 & Dulne & Jarczów & $25 \times 30$ & $\mathrm{C}$ \\
\hline 61 & Narol & Narol & $20 \times 20$ & $\mathrm{C}$ \\
\hline 62 & Lipsko & Narol & $100 \times 150$ & A \\
\hline 63 & Lipsko & Narol & $100 \times 100$ & $\mathrm{~A}$ \\
\hline 64 & Narol-Krupiec & Narol & $60 \times 150$ & A \\
\hline 65 & Jędrzejówka & Narol & $15 \times 15$ & $\mathrm{C}$ \\
\hline 66 & Stara Huta & Narol & $30 \times 50$ & C \\
\hline 67 & Wola Wielka & Narol & $3 \times 30$ & $\mathrm{C}$ \\
\hline 68 & Wola Wielka & Narol & $25 \times 30$ & $\mathrm{C}$ \\
\hline 69 & Kijasówka & Narol & $50 \times 100$ & $\mathrm{~A}$ \\
\hline 70 & Bełżec & Bełżec & $10 \times 10$ & $\mathrm{C}$ \\
\hline 71 & Łosiniec & Susiec & $70 \times 70$ & $\mathrm{C}$ \\
\hline 72 & Łosiniec & Susiec & $100 \times 150$ & A \\
\hline 73 & Majdan Sopocki Drugi & Susiec & $150 \times 200$ & A \\
\hline 74 & Susiec & Susiec & $50 \times 50$ & $\mathrm{~A}$ \\
\hline 75 & Huta Różaniecka & Narol & $30 \times 40$ & $\mathrm{C}$ \\
\hline 76 & Huta Różaniecka & Narol & $40 \times 40$ & A \\
\hline 77 & Lisie Jamy & Lubaczów & $15 \times 60$ & $\mathrm{~A}$ \\
\hline 78 & Lisie Jamy & Lubaczów & $25 \times 25$ & $\mathrm{C}$ \\
\hline
\end{tabular}

Explanations: A - active cemeteries, $\mathrm{C}$ - closed cemeteries. 


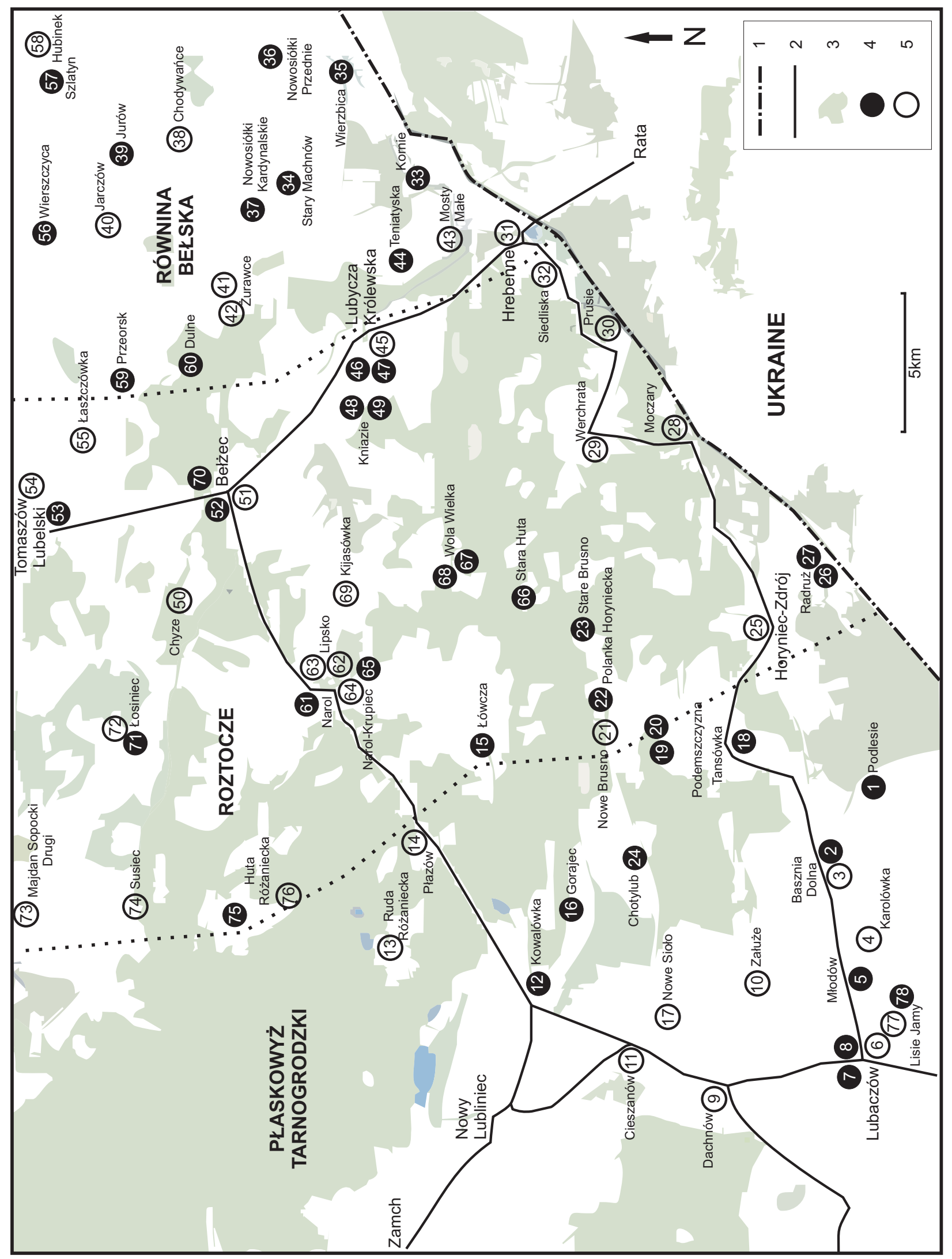

Fig. 1. Locations of the investigated cemeteries in the Roztocze region.

Explanations: 1 - state border; 2 - main roads; 3 - forest area; 4 - closed cemeteries; 5 - active cemeteries. Cemeteries are denoted with numbers as in Table 1. 


\section{RESULTS}

A list of families and species

The explanation of the manner of presentation of information in the "Material and Methods" chapter.

Equisetaceae: Equisetum arvense L. [Ap G 6] 3(II), 4, 6, 7, 9, 10 (II), 11(II), 14, 15, 17, 19, 21, 23, 25, 29, 30, 32, 40, 41, 45, 46, 47, 49, 51, 53(III), 54(II), 60, 63, 64, 72, 73, 74, 77; Equisetum sylvaticum L. [Ap G 1] 60;

Hypolepidaceae: Pteridium aquilinum (L.) Kuhn [Sp G 2] 4, 12, 20, 64, 74;

Aspleniaceae: Asplenium ruta-muraria L. [Ap H 18] 25, 28; Asplenium trichomanes L. [Ap H 18] 66 ;

Athyriaceae: Athyrium filix-femina (L.) Roth [Sp H 1] 10, 18, 20, 49; Cystopteris fragilis (L.) Bernh. [Sp H 18] 11; Matteucia struthiopteris (L.) Tod. [Sp H 1] $3,5,6,9,10,11,14,17,25,45,49,50,54,62,63$, 64, 68 (II), 73, 74, 77;

Aspidiaceae: Dryopteris carthusiana (Vill.) H. P. Fuchs [Sp H 8] 12 (II), 16, 18, 20, 24, 43, 44, 48, 60, 65, 66, 78; Dryopteris dilatata (Hoffm.) A. Gray [Sp H 1] 49, 66, Dryopteris filix-mas (L.) Schott [Sp H 2] $11,12,15,16,17,18,23,25,26,27,31,48,49$, $54,60,67$; 2] 24;

Polypodiaceae: Polypodium vulgare L. [Sp H

Pinaceae: Abies alba Mill. [Ap M 1] 28, 52, 70; Larix decidua Mill. [D M 2] 17, 25, 53, 78; $\underline{\text { Picea }}$

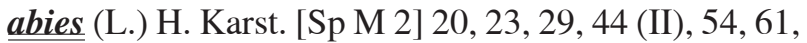
66 (II), 67, 77; Pinus sylvestris L. [Ap M 5] 8, 12, 13, 14, 15, 16 3, 17, 19 (II), 20, 21, 22, 23, 24, 28, 31, 42, 43 (II), 44 (II), 46, 47, 49, 51, 61 (II), 63, 64, 65 (II), 66 (II), 67 (II), 69, 70, 74, 78 (II); Tsuga canadensis (L.) Carričre [D M 19] 6;

Cupressaceae: Juniperus communis $\mathrm{L}$. [Sp N 5] 19, 43, 44, 65, 66;

Salicaceae: Populus alba L. [Ap M 7] 15, 39; Populus nigra L. [Ap M 7] 49; Populus termula L. [Ap M 2] 7, 16, 23, 46, 47 (II), 60, 64, 74; Salix alba L. [Ap M 7] 43, 53; Salix alba L. f. viteliana pendula Rehd. [D M 19] 6, 8; Salix caprea L. [Ap N 3] 4, 7, 76, 25; Salix triandra L. [Sp N 7] 62; 48, 53;

Juglandaceae: Juglans regia L. [D M 19]

Betulaceae: Alnus glutinosa (L.) Gaertn. [Sp M 6] 21; Betula pendula Roth [Ap M 2] 8 (II), 11, 16, 18 (II), 19 (II), 21, 28, 42, 44, 45, 46, 47, 49, 51, 53, 54, 60, 66 (II), 67 (II), 69 (II), 72, 74, 75;

Corylaceae: Carpinus betulus L. [Sp M 1] 1, 12, 24 (III), 27 (III), 49 (II), 52, 66, 75; Corylus avellana L. [Sp N 1] 16, 23, 26, 28, 29, 31, 36, 48, 49, 51, 60;

Fagaceae: Fagus sylvatica L. [Sp M 1] 16, 23, 44, 66, 67, 70; Quercus robur L. [Sp M 1] 1, 2, 5, 8, 9,
12 (III), 15, 16 (II), 17, 19 (II), 20, 21, 22, 23 (II), 24

(II), 25, 26, 27, 28, 29, 30, 31, 32, 38, 43, 44 (II), 46 (II), 51 (III), 52, 60 (II), 61, 63, 65, 66, 70, 75, 78 (III); Quercus rubra L. [Ken M 2] 70;

Ulmaceae: Ulmus laevis Pall. [Sp M 1] 7, 11, 12, 13, 34, 36, 37, 53, 56, 58(II);

Cannabaceae: Humulus lupulus L. [Sp H 7] 11, 25, 31, 34, 36, 51, 59;

Urticaceae: Uritca dioica L. [Ap H 3] 1, 2, 5, 6, 7, 8, 10, 12 (II), 14, 16, 17, 18, 20, 23, 24, 25, 27, 28, $29,31,32,33,34,35,36,37,39,41,42,44,46,47,48$, $49,51,53,54,56,57,58,59,60,62,63,64,67,69,71$, 73, 74, 75; Urtica urens L. [Arch T 16] 64;

Loranthaceae: Viscum album L. [Ap Ch 1] 25, 27, 59, 75; H 1] 59;

Aristolochiaceae: $\underline{\text { Asarum europaeum }}$ L. [Sp

Polygonaceae: Fallopia convolvulus (L.) Á. Löve [Arch T 16] 4, 6, 8, 11, 12, 14, 29, 33, 42, 43, 44, 49, 50, 51, 58, 60, 64, 76; Fallopia dumetorum (L.) Holub [Sp T 2] 2, 6, 7, 14, 15, 16, 25, 29, 30, 31, 33, 39, 42, 51, 54, 62, 63, 64, 68; Polygonum amphibium L. f. terrestre [Ap G 10] 45; Polygonum aviculare L. [Ap T 10] 4, 5, 6, 8, 9, 10, 11, 13, 17, 21, 25, 27, 29, 30, 31, 32, 35, 38, 40, 41, 42, 43, 46, 51, 54 (II), 55, 58, 62, 63, 68, 72, 74, 77; Polygonum bistorta L. [Sp G 8] 4; Polygonum hydropiper L. [Ap T 12] 77; Polygonum lapathifolium L. ssp. lapathifoium [Ap T 12] 6, Polygonum lapathifolium L. ssp. pallidum (With.) Fr. [Ap T 16] 3, 33; Polygonum minus Huds. [Ap T 12] 25, 51; Polygonum orientale L. [D T 19] 6, 10; Polygonum persicaria L. [Ap T 16] 41, 45, 51, 54, 72, 73, 74; Reynoutria japonica Houtt. [Ken G 16] 2, 11, 20, 25, 42, 45, 72; Rumex acetosa L. [Ap H 9] 2, 3, 4, 5, 7, 9; Rumex acetosella L. [Ap G 5] 4, 5, 16, 21, 22, 28, 30, 42, 43, 47, 61, 65, 66, 67, 69, 74, 76, 78; Rumex confertus Willd. [Ken H 14] 3, 6, 10, 32; Rumex crispus L. [Ap H 10] 29, 40, 54, 55, 64, 75; Rumex obtusifolius L. [Ap H 13] 9, 14, 23, 26, 27, 41, 49, 51, 53, 54, 58, 62, 68; Rumex thyrsiflorus Fingerh. [Ap H 14] $11,13,14,17,19,21,25,28,29,30,31,32,41$, $42,43,44,45,46,53,55,62,63,64,66,67,68,69,72$, 73, 74, 75, 76, 77, 78;

Chenopodiaceae: Atriplex hortensis L. [Ken T 16] 77; Atriplex nitens Schkuhr [Arch T 15] 54; Atriplex patula L. [Ap T 16] 7, 9, 14, 29, 34, 35, 38, 39, 40, 51, 54, 64; Chenopdodium album L. [Ap T 16] 3, $5,6,8,9,11,13,14,17,18,21,25,28,29,30,31,32$, 33 (II), 38, 40, 41, 42, 43, 45, 47, 50, 51, 54, 55, 58, 62, 63 (II), 64, 68, 71, 72, 73, 74, 76, 77; Chenopodium hybridum L. [Arch T 16] 33, 35, 58; Chenopodium polyspermum L. [Ap T 12] 31, 35; Chenopodium strictum Roth [Ken T 15] 73, 77; Fagopyrum esculentum Moench [D T 16] 54; Kochia scoparia (L.) Schrad. [Ken T 15] 3, 6, 9, 40; 
Amaranthaceae: Amaranthus caudatus L. [Ken T 16] 73, 77; Amaranthus chlorostachys Willd. [Ken T 16] 38; Amaranthus cruentus L. [Ken T 16] 6, 9, 10, 11, 17, 25, 29, 38, 40, 51, 54, 68, 74, 77; Amaranthus retroflexus $\mathrm{L}$. [Ken T 16] 9, 29, 33, 35, 40, 41, 58, 73; Celosia spicata L. [D T 19] 77;

Anacardiaceae: Rhus typhina L. [D M 16] 8, 50, 59, 64;

Portulaceae: Portulaca grandiflora Hook. [D $\mathrm{T}$ 19] 6; Portulaca oleracea L. [Ken T 16] 31; Caryophyllaceae: Arenaria serpyllifolia L. [Ap T 5] 31, 42, 47, 51, 72, 73, 76; Cerastium arvense L. s.s. [Ap H 9] 47, 53, 64; Cerastium biebersteinii DC. [D H 19] 5, 16, 25, 35; Cerastium holosteoides Fr. emend. Hyl. [Ap H 9] 3, 10, 14, 25, 26, 51, 53, 54, 69, 72, 73; Cucubalus baccifer L. [Sp H 3] 29, 39; Dianthus barbatus L. s.s. [Ken C 19] 1, 8, 14, 16, 21, 35, 47, 51; Dianthus deltoides L. [Ap H 2] 46; Gypsophila paniculata L. [Sp H 16] 50; Herniaria glabra L. [Ap H 5] 4, 42, 71, 76; Melandrium album (Mill.) Garcke [Ap H 14] 1, 2, 3, 5, $6,7,9,10,11,14,1516,17,19,21,22,24,25,27,28$, $30,31,32,3338,39,41,44,45,46,47,49,50,51,53$, 54, 55, 58, 62, 66, 68, 69, 71, 72, 73, 74, 75; Moehringia trinervia (L.) Clairv. [Sp H 2] 8, 12, 14, 18, 20, 22, 23, 24, 25, 26, 27, 29, 47, 48, 66, 71; Myosoton aquaticum (L.) Moench [Ap H 7] 35; Saponaria officinalis L. [Arch G 14] 2, 3, 6, 7, 10, 11, 14, 15, 17, 21, 22, 25, $28,30,31,32,35,36,38,40,43,44,45,46,47,49,51$, 54, 62, 63, 64, 69, 71, 73, 74, 76; Scleranthus annuus L. [Arch T 17] 25, 77; Scleranthus perennis L. [Ap H 5] 13, 21, 42, 43, 55, 69, 76; Silene armeria L. [D T 19] 3 , 4, 25, 54; Silene vulgaris (Moench) Garcke [Ap H 14] 13, 14, 16, 19, 21, 25, 28, 38, 62, 63, 64 (II), 67 (II), $69,72,73,74,75,76,77$; Spergula arvensis L. [Arch T 16] 3, 9, 10, 13, 25, 30, 50, 55 (II), 74, 77; Spergularia rubra (L.) J. Presl \& C. Presl [Ap T 5] 3, 4, 25, 54, 77; Stellaria graminea L. [Ap H 2] 31, 47; Stellaria holostea L. [Sp H 1] 12, 52; Stellaria media (L.) Vill. [Ap T 16] $3,4,6,9,11,14,21,25,27,31,32,33,34,35,40$, 44, 50, 51, 53, 58, 60, 62, 63, 64, 72, 73;

Ranunculaceae: Actea spicata L. [Sp H 1] 23; Aquilegia vulgaris L. [Sp H 1] 4, 9, 10, 15, 25, 32, 36, 38, 40, 44, 48, 49, 51, 68, 73, 74; Consolida ajacis (L.) Schur [D T 16] 11, 17, 35; Consolida regalis Gray [Arch T 17] 14, 38; Hepatica nobilis Schreb..[Sp H 1] 12, 35, 59; Ranunculus acris L. s.s. [Ap H 9] 3, 4, 5, $8,9,10,11,14,21,25,26,27,28,29,30,31,32,38$, $40,44,45,46,47,49,51,53,54,57,58,62,63,64$ (II), 67, 68, 71, 72, 73, 74, 76; Ranunculus auricomus $\mathrm{L}$. s.1. [Sp H 1] 22, 29; Ranunculus bulbosus L. [Ap H 4] 28, 45, 73; Ranunculus repens L. [Ap H 10] 14, 25, 26, 27, 51, 53, 54; Thalictrum minus L. [Sp H 4] 64; Trollius europaeus L. S.S. [Sp H 8] 51;

Berberidaceae: Berberis vulgaris L. [Ap N 4] 13; Mahonia aquifolium (Pursh) Nutt. [Ken N 19] 74;
Papaveraceae: Chelidonium majus L. [Ap H 3] $2,8,11,12,14,15,18,20,21,22,23,24,25,27,28$, $30,32,33,34,35,37,42,46,47,48,49,56,58,59,60$, 62, 63, 64, 66, 67, 69, 71, 73, 75, 76, 78; Eschscholtzia californica Cham. [D T 19] 6, 9; Papaver argemone L. [Arch T 17] 11; Papaver dubium L. [Arch T 17] 11, 14, 64, 76; Papaver rhoeas L. [Arch T 17] 11, 54, 64, 72;

Fumariaceae: Fumaria officinalis L. [Arch T 16] 51, 54; Fumaria vaillantii Loisel. [Arch $T$ 14] 64;

Brassicaceae: Alliaria petiolata (M. Bieb.) Cavara \& Grande [Ap T 3] 15; Alyssum alyssoides (L.) L. [Ap T 5] 13; Arabidopsis thaliana (L.) Heynh. [Ap T 17] 3, 4, 6, 11, 21, 30, 45, 51, 53, 54, 62, 63, 64, 72, 73; Arabis alpina L. [Sp H 2] 77; Armoracia rusticiana P. Gaertn., B. Mey. \& Scherb. [Arch H 13] 6, 14, 45, 55, 57, 62, 64; Berteroa incana (L.) DC. [Ap T 14] 5, $8,11,16,21,25,28,29,30,32,42,43,45,46,47,50$, $62,63,64,67,68,69,72,73,75,76$; Brassica napus L. [Ken T 19] 40; Bunias orientalis L. [Ken H 14] 38; Capsella bursa-pastoris (L.) Medik. [Arch T 16] 3, 6, $9,10,25,28,30,33,35,38,40,41,45,46,51,53,58$, 62, 63, 64 (II), 68, 72, 73 (II), 74, 77; Cardaminopsis arenosa (L.) Hayek [Sp H 9] 25, 31 (II), 32, 54, 74; Descurainia sophia (L.) Webb ex Prantl [Arch T 16] 6, 11; Erysimum cheiranthoides L. [Ap T 16] 3, 6, 11, 14, 25, 29, 35, 40, 46, 47, 51, 54, 62, 63, 64, 73; $\underline{\text { He}-}$ speris matronalis L. [Ken H 16] 35, 36, 59; Lepidium densiflorum Schrad. [Ken T 15] 8, 55; Raphanus raphanistrum L. [Arch T 16] 55; Rorippa palustris (L.) Besser [Ap T 12] 6, 9, 10, 21, 25, 54, 64 2, 72; Sinapis arvensis L. [Arch $\mathrm{T}$ 16] 54; Thlaspi arvense $\mathrm{L}$. [Arch T 16] 35, 51;

Crassulaceae: Jovibarba sobolifera (Sims) Opiz [Sp H 5] 72; Sedum acre L. [Ap H 5] 4, 5, 10 , $11,13,28,29,43,50,55,73$ (II), 74, 76 (II), 77; $\underline{\underline{S e}-}$ dum album $\mathrm{L}$. [Ken $\mathrm{H}$ 16] 73; Sedum maximum (L.) Hoffm. [Sp G 2] 5, 10, 12, 46, 74, 78; Sedum sexangulare L. [Sp H 5] 5, 16, 32, 43, 45, 54, 67, 72; $\underline{\text { Sedum }}$ spectabile Boreau [D H 16] 3, 9, 62; Sedum spurium M. Bieb. [Ken H 16] 5, 9, 16, 41, 43, 76; Sempervivum tectorum L. [D H 19] 44;

Saxifragaceae: Bergenia cordifolia (Haw.) Sternb. [D H 19] 29, 42;

Hydrangeaceae: Philadelphus coronarius L.

[D N 19] 16, 22, 39, 59;

Grossulariaceae: Ribes spicatum E. Robson [Sp N 1] 15, 24, 57, 60, 66; Ribes uva-crispa L. [Ken N 2] 23, 29, 36, 44, 48, 51, 57, 78;

Rosaceae: Agrimonia eupatoria L. [Ap H 4] 14, 28, 38, 40, 51, 57, 59, 67; Alchemilla monticola Opiz [Ap H 8] 53 (II); Amelanchier spicata (Lam.) K. Koch [Ken N 2] 65; Cerasus avium (L.) Moench [Ken M 19] 7, 8, 11, 15, 23, 31, 39, 52, 57, 67, 78; Cerasus vulgaris Mill. [D M 19] 8 (II), 22, 24, 34 (II), 38, 48, 
57 (II), 59, 71; Crataegus monogyna Jacq. [Ap N 1] 7, 11, 14 3, 19, 24, 28, 29, 36, 47, 48 (II), 49, 59; Fragaria $\times$ ananassa Duchesne [D H 19] 73; Fragaria moschata Duchesne [Ap H 2] 1, 2, 12, 21, 25, 29, 47, 48, 57, 67; Fragaria vesca $\mathrm{L}$. [Sp H 2] 18, 26, 28, 44, 49, 65, 74; Fragaria viridis Duchesne [Ap H 4] 4, 10, 14, 23, 27, 31, 32, 33, 38, 41, 53, 67, 69, 71, 74, 75; Geum urbanum L. [Ap H 3] 8, 12, 15, 18, 22, 23, 24, 26, 27, 29, 33 (II), 34, 36, 37, 38, 39, 46, 48, 49, 51, 52, 53, 57, 59, 60, 66, 67, 75; Malus domestica Borkh. [D M 19] 36, 37, 41, 46, 49, 50, 53, 57, 59, 67; Malus sylvestris Mill. [Ap M 1] 15; Padus avium Mill. [Sp M 1] 2, 12, 15, 18, 19, 24, 36, 37, 42, 44, 48, 60; Padus serotina (Ehrh.) Borkh. [Ken T 15] 19, 73; Potentilla anserina L. [Ap H 10] 3, 4, 6, 10, 40, 51, 53, 54, 73; Potentilla argentea L. s.s. [Ap H 14] 47; Potentilla collina Wibel s.s. [Ap H 4] 4, 25, 28, 30, 42, 43, 44, 45, 46, 67, 68, 71, 72, 73, 74, 76; Potentilla reptans L. [Ap H 10] 35, 38, 40; Prunus cerasifera Ehrh. [Ken M 16] 8, 15, 39, 51, 57; Prunus spinosa L. [Ap N 4] 14, 57, 59, 67; Pyrus pyraster (L.) Burgsd. [Ap M 1] 7, 8, 18, 20, 31, 33, 48, 53, 59, 65; Rosa canina L. [Ap N 4] 14, 15, 16, 22, 26, 28, 29, 38, 47, 48, 51, 59, 62, 64, 68, 73, 74, 75; $\underline{\text { Rosa }}$ xfrancofourtana Münchh. [Ken N 16] 40 54, 57, 73, 76; Rosa majalis Herrm. [Ken N 19] 5, 11, 14, 22, 25, 32, 43, 57, 62, 63, 64, 73; Rosa rugosa Thunb. [Ken N 16] 3, 21, 28, 41, 47, 74, 76; Rosa sherardii Davies [Ap N 4] 74; Rubus caesius L. [Ap H 13] 1, 4, 7, 9, 11, $14,15,25,29,31,32,33,34,37,38,41,46,47,48,49$, 51, 57, 59, 63, 73; Rubus hirtus Waldst. \&Kit. [Sp N 1] 20, 49; Rubus idaeus L. [Sp N 2] 1, 2, 12, 15, 16, $20,21,22,23,24,25,26,28,29,31,32,42,43,44,46$, 47, 48, 49, 51, 53, 60, 61, 63, 64, 65, 66, 67, 69, 71, 72, 74, 75, 78; Rubus nessensis Hall [Sp N 2] 48; Rubus ortostachys G. Braun [Ap N 1] 48; Rubus pedemontanus Pinkw. [Sp N 1] 60, 65, 66; Rubus plicatus Weihe \& Nees [Sp N 2] 12, 13, 19, 20, 22, 24, 31, 42, 43, 53, 66, 67, 71, 78; Rubus rudis Weihe [Sp N 1] 15, 23; Sanguisorba minor Scop. s.s. [Sp H 4] 38; $\underline{\text { Sorbaria }}$ sorbifolia (L.) A. Braun [Ken N 16] 8, 25, 31, 43, 44, 64; Sorbus aucuparia L. emend. Hedl. [Sp M 2] 12, 15 , 16 (II), 18, 19 (II), 20 (II), 22, 23, 24 (II), 26 (II) 43, 44, 45, 46, 48, 49, 51 (II), 52, 53 (II), 54, 60, 61, 65 (II), 66 (II), 67, 69, 72, 74 (II), 78 (II); Spiraea chamaedryfolia L. emend. Jacq. [Ken N 16] 12, 22; Spiraea salicifolia L. [Sp N 16] 1, 12, 25, 44, 46;

Fabaceae: Astragalus cicer L. [Ap H 4] 38; Astragalus glycyphyllos L. [Ap H 2] 14, 21, 22, 29, 51, 52, 73; Coronilla varia L. [Ap H 4] 7, 13, 14, 21, 22, 25, 29, 38, 40, 45, 51, 57, 62, 64, 73, 74, 75; Lathyrus pratensis L. [Ap H 9] 14, 41; Lembotropis nigricans (L.) Griseb. [Ap N 5] 13, 43, 69, 78; Lotus corniculatus L. [Ap H 9] 4, 5, 14, 25, 38, 51; Lupinus polyphyllus Lindl. [Ken H 19] 2, 3, 4, 5, 6, 9, 10, 11, 21, 25, 30, 40, 41, 45, 50, 51, 54, 63, 68, 72, 73, 74, 76; Medicago falcata L. [Ap H 4] 7, 14 (II), 29, 38, 40, 41, 62, 63, 64, 75; Medicago lupulina L. [Ap H 9] 6, 11, 14, 25, 29, 30, 35, 38 (II), 41, 43 (II), 45, 51, 53, 62, 63, 64, 68, 73, 74; Medicago xvaria Martyn [Ap H 14] 38; Melilotus alba Medik. [Ap T 15] 3, 26, 40, 43; Melilotus officinalis (L.) Pall. [Ap T 15] 14, 21, 35, 38, 40, 51, 75; Robinia pseudoacacia L. [Ken M 16] 1, 2 (II), 5, 6, 7, 8 (II), 10, 12 (II), 13, 15, 17, 21, 24 (II), 28, 30, 32, 33, 39 (III), 42, 43, 44 (II), 46 (II), 48 (II), 49 (II), 51, 56 (II), 58 (II), 59 (II), 60, 62 (II), 63 (II), 64, 67, 68, 69 (II), 75, 76; Sarothamnus scoparius (L.) W. D. J. Koch [Ken Ch 5] 69; Trifolium arvense $\mathrm{L}$. [Ap T 5] 7, $11,12,14,17,19,21,25,28,33,45,46,50,51,55,62$, 63, 64, 76; Trifolium campestre Schreb. [Ap T 9] 45, 71, 72; Trifolium hybridum L. [Ap T 9] 41; Trifolium medium L. [Ap H 2] 1, 10, 11, 14, 22, 29, 62, 63, 64, 72, 73, 74; Trifolium pratense L. [Ap H 9] 3 (II), 4, $6,9,10,11,14,17,21,25,27,30,31,32$ (II), 38, 40, $41,45,51,53,54,58,62,63,64,67,72,73,74,76$; Trifolium repens L. [Ap H 10] 3, 4, 9, 10, 14, 17, 25, $26,27,28,29,30,32,35,38,40,41,47,50,51,53,55$, 62, 67, 69, 72, 73, 74, 76; Vicia angustifolia L. [Ap T 17] 11, 40, 45; Vicia carcca L. [Ap G 8] 1, 2, 3, 4, 9, $10,11,14,16,19,21,25,29,30,38,40,46,51,53,54$, 62, 63, 64, 68, 72; Vicia dumetorum L. [Ap H 3] 54; Vicia hirsuta (L.) Gray [Arch T 17] 33, Vicia villosa Roth [Arch T 17] 33, 73;

Oxalidaceae: Oxalis acetosella L. [Sp H 2] 20, 23, 48, 49, 60; Oxalis corniculata L. [Ken H 16] 10, 25, 35; Oxalis dillenii Jacq. [Ken T 16] 64, 72; Oxalis fontana Gunge [Ken T 16] 2, 3, 4, 5, 6, 9, 10, 11, 16, $21,24,25,26,27,28,29,30,31,32,402,45,46,49$, $50,51,54,62,63,64,68,71,72,73,74$;

Geraniaceae: Erodium cicutarium (L.) L'Hér. [Ap T 16] 55, 73; Geranium molle L. [Ken T 14] 31, 33; Geranium phaeum L. [Sp H 2] 12, 14, 26, 27, 29, 30; Geranium pratense L. [Ap H 9] 9 (II), 21, 38, 41, 45, 46, 51, 54, 57, 58, 63; Geranium pusillum Burm. F. ex L. [Arch T 16] 11, 14, 30, 33, 35, 38 (II), 45, 50, $51,53,62,72,73,74$; Geranium robertianum L. [Sp T 3] 2, 15, 16, 23, 24, 25, 28, 40, 49, 60, 66; Geranium sibiricum L. [Ken H 19] 54;

Euphorbiaceae: Euphorbia cyparissias L. [Ap H 4] 3, 4, 8, 14, 15, 16, 21, 22, 25, 26, 28, 29, 30, 38, 40 (II), 43, 44, 46, 47, 51, 53, 57, 63, 64, 66, 68, 69, 73, 74, 75, 76 (II), 77 (II); Euphorbia esula L. [Ap H 14] 39; Euphorbia helioscopia L. [Arch T 16] 35, 38, 40; Euphorbia marginata Pursh [Ken T 16] 3, 4, 6, 11, 17, $21,32,35,38,40,45,51,54,55,62,63,64,68,72,73$, 76; Euphorbia peplus L. [Arch T 16] 63;

Aceraceae: Acer negundo L. [Ken M 3] 7, 56; Acer platanoides L. [Ap M 1] 9, 11, 15, 18 (II), 24, 27, 36 (II), 47, 49, 52 (II), 54, 64; Acer pseudoplatanus L. [Ap M 1] 5, 10, 15 (II), 21 (II), 26 (II), 30, 31, 35, 36, 39, 43, 51, 54, 57 (II), 60, 62, 66 (II), 69; 
Hippocastanaceae: Aesculus hippocastanum L. [Ken M 19] 15, 49 (II);

Balsaminaceae: Impatiens balsamina L. [Ken $\mathrm{T}$ 7] 3; Impatiens glandulifera Royle [Ken T 7] 6, 9, 11; Impatiens parviflora DC. [Ken T 3] 6, 9, 11, 14, 15, 31, 33, 44, 49, 61, 73, 74;

Celastraceae: Euonymus europaea L. [Ap N 1] $7,11,12,14,15,18,26,27,33,34,35,36,37,38$, 39, 41, 46, 47, 48, 58, 59, 60, 64, 73, 75; Euonymus verrucosa Scop. [Ap N 1] 53;

Rhamnaceae: Frangula alnus Mill. [Sp N 3] 11, 12, 16, 20, 23, 28, 31, 32, 42, 43, 44, 61, 66 (II), 67, 69, 74 (II), 75, 78; Rhamnus catarthica L. [Sp N 1] 7, 29, 31, 34, 36, 46, 47, 48, 51, 53, 56, 59, 66;

Vitaceae: Parthenocissus quinquefolia (L.) Planch. In A. \& C. DC. [Ken N 16] 11, 16, 21, 29, 73;

Tiliaceae: Tilia cordata Mill. [Ap M 1] 1, 2, 5, 6, 10, 11, 12 (III), 14, 15 (II), 18 (II), 19, 20 (III), 21, 22 (II), 23, 24 (II), 25, 26 (III), 27 (III), 28, 29, 30, 36 (II), 37, 40, 44, 47, 48 (II), 51, 52 (II), 53, 54, 56 (II), 57, 58, 59, 60 (II), 62, 63, 66, 74, 75 (II); Tilia хеuchlora K. Koch [D M 19] 74; Tilia platyphyllos Scop. [Ap M 1] 14, 15, 66, 67, 69;

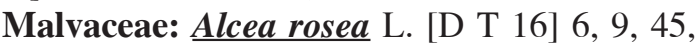
54; Lavatera thuringiaca $\mathrm{L}$. [Ken H 4] 34, 58; Malva alcea L. [Ap H 4] 7, 14, 29, 41, 45, 51, 65; Malva neglecta Wallr. [Arch T 14] 11, 30, 35, 45; Malva pusilla Sm. [Arch T 14] 35; Malva sylvestris L. [Arch T 14] 35, 38, 41, 45, 64;

Oleaceae: Fraxinus excelsior L. [Ap M 1] 10, 15, 23, 28, 29, 33, 34 (II), 35, 36 (II), 37, 38 (II), 39 (III), 40, 41, 46 (II), 47, 48, 53, 54, 56, 57 (II), 58, 59 (II), 60, 62, 63, 75 (II), 76; Fraxinus excelsior L. 'Pendula' [D M 19] 6, 64; Fraxinus pensylvanica Marshall [Ken M 19] 76; Ligustrum vulgare L. [D N 16] 12, 16, 26, 48; Syringa vulgaris L. [Ken N 16] 3, 10, 12, 14, $15,16,19,21,22,23,24,28,29,30,34,36,37,39$ (II), $41,43,44,46,47,49$ (II), 51, 54, 56, 57, 58, 59 (II), 60, $62,63,64,66,67,68,69,71,75,78$;

Guttiferae: Hypericum maculatum Crantz [Sp $\mathrm{H}$ 2] 12; Hypericum perforatum $\mathrm{L}$. [Ap H 2] 1, 2, 5, 7 , $10,11,13,14,15,16,17,19,21,22,23$ (II), 25, 26, 27, $28,29,33,36,38,41,42,43,44,46,47,50,51,53,57$, $60,62,63,64,65,66,69,71,72,73,75,76$;

Violaceae: Viola arvensis Murray [Arch T 17] 4, 5, 8, 13, 16, 28 (II), 43, 44, 45, 72; Viola canina L. s.s. [Ap H 2] 5, 13, 25, 28, 42, 43, 44, 69, 71; Viola collina Besser [Ap H 1] 26; Viola odorata L. [Ken H 3] 2, 6, 12, 14, 21, 22, 23, 24, 27, 33, 35, 36, 37, 38, 39, 44, 47, 48, 52, 54, 56, 57 (II), 58, 59, 62, 63; Viola reichenbachiana Jord. ex Boreau [Sp H 1] 14, 20, 44, 52, 54, 60, 65; Viola tricolor L. s.s. [Ap H 5] 21, 22, 30, 31, 38, 41, 63, 73; Viola $\times$ wittrockiana Hort. [D T 19] 35, 38, 49, 54;

Cucurbitaceae: Echinocystis lobata (F. Michx.) Torr. \& A. Gray [Ken T 7] 45;
Onagraceae: Chamaenerion angustifolium (L.) Scop. [Ap H 2] 1, 2, 21, 22, 28, 29, 31, 46, 51, 65, 67, 74; Epilobium adanatum Griseb. [Ap H 12] 22, 28, 29, 30, 35; Epilobium ciliatum Raf. [Ken H 12] 13, 17, 54, 55, 64; Epilobium montanum L. [Ap H 1] 12 (II), 15, 16, 18, 20, 22, 25, 26, 28, 29, 38, 49, 51; Oenothera biennis L. s.s. [Ap T 14] 6, 11, 13, 14, 22, $25,28,30,32,33,40,43,45,46,50,51,54,55,62,63$, 64, 72, 73, 74, 76, 77; Oenothera glazioviana Micheli in Mart. [D T 19] 21;

Cornaceae: Cornus sanguinea $\mathrm{L}$. [Sp N 1] 14, 36, 38, 57; Cornus sericea L. [Ken N 16] 53; 54,74

Araliaceae: Hedera helix L. [Ap H 1] 41,

Apiaceae: Aegopodium podagraria L. [Sp H 1] $1,2,3,6,7,8,9,10,11,12,14,16,17,18,21,22,23$, $24,25,26,27,28,29,30,31,32,33,34,35,36,37,38$, $39,40,41,45,46,47,49,51,52,53,54,55,56,57,58$ (II), 59, 60, 62, 63, 64, 67, 68, 71, 72, 73, 74, 75, 76; Aethusa cynapium L. [Arch T 16] 11, 14, 25, 54, 72, 73; Anethum graveolens L. [D T 16] 14, 35, 74; Anthriscus sylvestris (L.) Hoffm. [Ap H 3] 4, 9, 10, 14, 28, 29, 30, 31, 38, 41, 43, 45, 46, 47, 48 (II), 51, 52, 53, 56, $58,60,63,71,73,75$; Chaerophyllum aromaticum $\mathrm{L}$. [Sp H 3] 29, 37, 51, 57, 58; Chaerophyllum bulbosum L. [Ap T 14] 37, 41, 47, 67; Chaerophyllum temulum L. [Ap T 3] 12, 15, 18, 25, 27, 37, 48, 49, 69; Daucus carota L. [Ap T 9] 2, 3, 19, 22, 25, 28, 38, 41, 44, 45, 51, 57; Eryngium planum L. [Ap H 4] 38(II), 40, 63, 68; Falcaria vulgaris Bernh. [Ap H 4] 38; $\underline{\text { Heracleum }}$ mantegazzianum Sommier \& Levier [Ken H 16] 34 (II); Heracleum sibiricum L. [Ap H 9] 17, 25, 40, 41, 46, 49, 51, 53, 54, 63, 64, 73; Heracleum sphondylium L. s.s. [Ap H 9] 9, 10, 11, 45; Pastinaca sativa L. s.s. [Ap H 9] 3, 38, 46, 51; Peucedanum oreoselinum (L.) Moench [Sp H 4] 30, 43; Pimpinella saxifraga L. [Ap H 9] 3, 8, 9, 10, 11, 14, 19, 21, 25, 29, 38, 40, 41, 44, 45, 46, 47, 49, 51, 53, 57, 62, 63, 64, 68 (II), 72 (II), 73, 74, 75, 76; Torilis japonica (Houtt.) DC. [Ap T 3] $7,14,15,16,18,19,22,23,25,26,27,29,36,37,38$, 42, 45, 46, 47, 48, 49, 69, 75;

Ericaceae: Calluna vulgaris (L.) Hull [Sp Ch 5] 43, 44, 65, 74; Vaccinium myrtillius L. [Sp Ch 5] 16, 20, 44, 61, 66, 70, 74, 78; Vaccinium vitis-idaea $\mathrm{L}$. [Sp Ch 5] 43, 44, 74;

Primulaceae: Anagallis arvensis L. [Arch T 16] 3, 50, 51, 54; Lysimachia nummularia L. [Sp H 1] 8, 9, 11, 14, 17, 26, 33, 35 2, 36, 48, 54; Lysimachia vulgaris L. [Sp H 7] 3, 4, 16, 17, 25; Primula veris $\mathrm{L}$. [Sp H 2] 36;

Apocynaceae: Vinca minor L. [Sp Ch 1], 2, 6, $8,10,11,12,14,15,16,18,19,20,21,22,23,24,25$, $26,27,28,29,30,31,33,34,35,36,37,38,39,41,42$, $43,46,47,48,49,51,57,59,60,62,63,66,67,68,69$, $71,73,74,75,76$; 
Asclepiadaceae: Asclepias syriaca L. [Ken H 16] 11, 45;

Rubiaceae: Cruciata glabra (L.) Ehrend. [Sp H 1] 30, 36, 38, 41, 43, 46, 47, 48, 49, 64; Galium aparine L. [Ap T 3] 27, 30, 35, 37, 44, 47, 48, 49, 59, 60, 72; Galium mollugo L. s.s. [Ap H 9] 1, 3, 5, 7, 8, 11, 12, 13, 14, 15, 19, 24, 27, 28, 29 (II), 30, 38, 40, 41, 45, 46, 49, 51, 53, 57, 62, 63, 65, 66, 74 (II) , 75, 76; Galium odoratum (L.) Scop. [Sp H 1] 36, 59; Galium spurium $\mathrm{L}$. [Arch T 17] 35; Galium verum L. s.s. [Ap H 9] 31, 32, 40, 57;

Polemoniaceae: Phlox paniculata L. [D H 19] 29; Phlox subulata L. [D H 19] 1, 72;

Convolvulaceae: Convolvulus arvensis L. [Ap G 14] 3, 6, 11, 14, 17, 25, 33, 34, 38, 40, 41, 45, 51, 53, 54, 57, 62, 64, 72, 73 (II), 76, 77;

Boraginaceae: Anchusa officinalis L. [Ap H 14] 6, 30, 41, 46, 62, 63, 64, 73; Echium vulgare $\mathrm{L}$. [Ap T 14] 21, 77; Myosotis arvensis (L.) Hill [Arch T 17] 30, 31, 32, 33, 35, 46, 51 2, 54, 63, 72; Myosotis stricta Link ex Roem. \& Schult. [Ap T 17] 4, 25; $\underline{\text { My- }}$ osotis sylvatica Ehrh. Ex Hoffm. [Sp T 1] 8, 35; $\underline{\underline{\text { Pul- }}}$ monaria obscura Dumort. [Sp H 1] 52; Symphytum officinale L. [Sp H 7] 26, 31, 62;

Lamiaceae: Acionos arvensis (Lam.) Dandy [Ap T 5] 75; Ajuga reptans L. [Sp H 1] 3, 38, 41, 45, 48, 52, 54, 64, 72, 73, 74; Ballota nigra $\mathrm{L}$. [Arch $\mathrm{H}$ 14] $26,33,34,35,36,37,38,39,47,48,56,58,59$, 60, 67, 75; Clinopodium vulgare L. [Sp H 2] 38, 57; Elsholtzia ciliata (Thunb.) Hyl. [Ken T 16] 73; Galeopsis pubescens Besser [Ap T 2] 1, 2, 7, 8, 9, 11, 12 (II), 13, 14, 15, 16, 18, 20, 21, 22, 23, 24, 25, 26, 27, $28,29,30,31,33,34,37,40,41,42,43,44,46,47,48$, $49,50,51,53,54,56,58,60,61,63,64,65,66,67$, 69, 73; Galeopsis tetrahit L. [Ap T 2] 12, 16, 18, 27, 51; Glechoma hederacea L. [Ap H 3] 5, 9, 11, 12, 14, $16,17,18,19,22,23,24,25,26,27,28,29,30,33$, $38,41,44,47,48,49,53,58,60,64,66,68,72,74$; Lamium album L. [Arch H 3] 2, 5, 9, 10, 11, 15, 27, 24, 25, 29, 31, 32, 33, 34, 35, 41, 44, 46, 47, 48, 49, $53,54,58,62,67,68,72$; Lamium argentatum $[\mathrm{D} \mathrm{H}$ 1] 12, 52; Lamium purpureum L. [Arch T 16] 11, 35, 38, 41; Leonurus cardiaca L. [Arch H 14] 7, 22, 27, 33, 34, 37, 52, 56; Mentha arvensis L. [Ap G 12] 30; Nepeta grandiflora $M$. Bieb. [D H 19] 58; Origanum vulgare L. [Ap H 2] 41; Prunella vulgaris L. [Sp H 9] 45, 46, 54, 57; Salvia verticillata L. [Ap H 16] 14, 38, 40, 58; Stachys sylvatica L. [Sp h 1] 23, 36; Thymus pulegiodes L. [Sp Ch 4] 3, 6, 19, 21, 25, 54, 75; Thymus serpyllum L. emend. Fr. [Ap Ch 5] 5, 43, 44, 69, 74, 76;

Solanaceae: Hyoscyamus niger L. [Arch T 14] 35; Lycium barbartum L. [Ken N 14] 7; Lycopersicon esculentum Mill. [D T 19] 9, 11, 54; Petunia xatkinsiana [D. Don 16] 77; Physalis alkekengi L. [Ken G
16] 25; Solanum dulcamara L. [Sp Ch 7] 24, 78; Solanum nigrum $\mathrm{L}$. emend. Mill. [Arch T 16] 35, 58;

Scrophulariaceae: Antirrhinum majus L. [D T 19] 11, 40; Euphrasia stricta D. Wolff ex J. F. Lehm. [Sp T 8] 25, 28; Linaria vulgaris Mill. [Ap G 2] 1, 2 , 10, 13, 19, 21, 22, 28, 31, 32, 42, 43, 44, 64, 65, 71, 73, 74, 76, 78; Melampyrum arvense L. [Ap T 4] 38, 59; Melampyrum pratense L. [Sp T 2] 44; Odontites serotina (Lam.) Rchb. s.s. [Sp T 10] 57; Scrophularia nodosa L. [Sp H 1] 1, 12, 18, 22, 27, 47, 48, 69; Verbascum densiflorum Bertol. [Ap T 14] 27, 28, 33, 43; Verbascum lychnitis L. [Ap T 5] 6, 29, 30, 41, 63, 66, 69, 75, 76; Verbascum nigrum L. [Ap T 2] 1, 9, 11, 12, 16, 17, 19, 28, 38, 41, 73; Verbascum phlomoides L. [Ap T 14] 6, 7, 11, 16, 22, 27, 29, 31, 41, 46, 54, 55; Verbascum thapsus L. [Ap H 14] 14; Veronica arvensis L. [Ap T 17] 3, 11, 25, 31, 51, 54, 64, 68, 72, 73; Veronica chamaedrys L. S.S [Ap H 9] 1, 2, 4, 5, 10, $11,12,14,15,16,18,19,21,23,24,25,26,27,28$, $29,30,31,33,36,38,41,42,44,45,46,48,49,51$, $52,53,62,66,67,68,71,72$ (II), 74, 75, 78; Veronica dillenii Crantz [Ap T 5] 43; Veronica officinalis L. [Ap H 2] 4, 12 (II), 16, 19, 22, 24, 44, 65, 66, 69, 71, 78; Veronica persica Poir. [Ken T 16] 35, 38, 40; Veronica polita $\mathrm{Fr}$. [Arch $\mathrm{T} 16$ ] 54, 72, Veronica verna L. [Ap T 5] 28;

Plantaginaceae: Plantago arenaria Waldst. \& Kit. [Ap H 12] 13, 76; Plantago intermedia Gilib. [Ap H 12] 72; Plantago lanceolata $\mathrm{L}$. [Ap H 10] 3, 4, 5, 9, $11,13,14,19,21,25,26,28,30,31,32,38,40,41,42$, $45,50,51,53,54,55,62,63,64,67,68,69,72,73,74$, 76, 77; Plantago major L. s.s. [Ap H 10] 4, 9 (II), 10, 13, 14 (II), 17, 23, 25, 27, 28, 29, 30, 35, 38, 40, 49, 51, 55, 64, 68, 71, 72, 73, 74; Plantago media L. [Ap H 9] 38, 40, 57;

Caprifoliaceae: Sambucus ebulus L. [Ap H 3] 67; Sambucus nigra L. [Ap N 3] 1, 2, 7, 14, 15, 18, 22, 24, 26, 27 (II), 29, 33, 34, 35, 36, 37 (II), 39, 48, 56, 57, 59, 60; Symphoricarpos albus (L.) S. F. Blake [Ken N 16] 22, 23, 24, 33, 36, 46, 47, 49, 57, 73, 74; Viburnum opulus L. [Sp N 7] 1, 16, 18, 20, 22, 23, 38, 47, 72, 75;

Dipsacaceae: Knautia arvensis (L.) J. M. Coult. [Ap H 2] 1, 3, 4, 7, 11, 12, 14, 19, 21, 25, 28, 29, $30,31,32,38,40,45,49,51,57,62,63,64,65,66$, $68,71,72,73,75,76$; Scabiosa ochroleuca L. [Sp H 4] 25, 38, 40;

Campanulaceae: Campanula patula L. S.S. [Ap H 9] 1, 7, 19, 25, 28; Campanula rapunculoides L. [Ap H 1] 14, 25; Campanula rotundifolia L. [Ap H 2] 32, 65, 74; Campanula trachelium L. [Sp H 1] 1, 9, 14, 29, 30, 36, 48, 52, 64; Jasione montana L. [Ap T 5] 14, 21, 22, 25, 31, 55, 76, 77;

Asteraceae: Achillea millefolium L. s.s. [Ap G 9] 3(II), 4(II), 5, 6, 9, 10, 11, 13, 14, 17, 19, 21, 25, 26, 
27, 28, 29, 30, 31, 32(II), 33, 38, 40, 41, 42, 43, 44, 45, 50, 51, 53, 54, 55, 58(II), 62, 63, 68, 69, 71, 72, 73, 74, 76, 77; Anthemis arvensis L. [Arch T 17] 3; Arctium lappa $\mathrm{L}$. [Ap T 13] 2, 9, 14, 22; Arctium tomentosum Mill. [Ap T 13] 37, 39, 51; Artemisia absinthium L. [Arch H 14] 22, 31, 73; Artemisia annua L. [Ken T 16] 58; Artemisia campestris L. [Ap H 5] 3, 13, 14, 19, 21, 28, 43(II), 55(II), 72, 76(II), 77; Artemisia ludoviciana Nutt. [D H 19] 4; Artemisia vulgaris L. [Ap H 13] 1, 2, $3,6,7,8,9,10,11,12,14,17,19,21,22,25,28,29$, 30, 33, 4, 38(II), 39, 40, 41, 43, 45, 46, 47, 51, 53, 54, 56, 7, 58, 62, 63, 64, 67(II), 69, 71, 72, 73, 74, 75, 77; Aster novi-belgii L. [Ken H 16], 2(III), 3(II), 8, 14, 15, 21(II), 25, 28, 29(II), 31(II), 46, 49, 51, 54, 57, 62, 63, 64, 67, 68, 73, 74, 75(III), 76; Aster xsalignus Willd. [Ken H 7] 10, 17, 63, 68; Bellis perennis L. [Sp H 9] 40, 50, 51, 54, 64, 68, 72; Bidens frondosa $\mathrm{L}$. [Ken T 12] 24, 41; Bidens tenuifolia Labill [D T 16] 3, 11, 25, 32; Bracteantha bracteata (Vent.) Anders. \& Haegi [D $\mathrm{T}$ 16] 11; Calendula officinalis L. [D T 16] 6, 9, 10, 11, 17, 21, 35, 73; Carduus acnathoides L. [Arch T 14] 7; Carduus crispus L. [Ap T 13] 33, 34, 45; Centaurea mollis Waldst. \& Kit. [Sp H 8] 10, 11, 54; Centaurea scabiosa L. [Ap H 4] 38, 40; Centaurea stoebe L. [Ap H 5] 14, 21, 25, 76, 77; Chamomilla suaveolens (Pursh) Rydb. [Ken T 10] 3, 9, 14, 51, 54, 63, 64; Chondrilla juncea $\mathrm{L}$. [Ap H 5] 13, 14; Cichorium intybus L. [Arch H 14] 14, 38, 51; Cirsium arvense (L.) Scop. [Ap G 13] 3, 6, 7, 10, 31, 33, 38, 40, 41, 51, 53(II), 54, 63; Cirsium rivulare (Jacq.) All. [Sp H 8] 54; Cirsium vulgare (Savi) Ten. [Ap T 13] 27, 38, 40, 43; Conyza canadensis (L.) Cronquist [Ken T 15], 2, 3(II), 5, 6(II), 9(II), 10(III), 11(III), 12, 13, 14, 15, 16, 17, 18, 19, 21, 22, 23, 24, 25, 26, 27, 28(II), 29(II), 30, 31, 32(II), 33, 34, 38, 40, 41, 42(IV), 43, 44, 45(II), 46(II), 47, 50(II), 51, 53, 54(III), 55(II), 58, 62(II), 63(II), 64(II), 68, 69, 71, 72(II), 73, 74, 75, 76, 77(II); Coreopsis grandiflora R. Hogg ex Sweet [D H 16] 32; Cosmos bipinnatus Cav. 4(II), 6(II), 9, 10(II), 11, 14, 17, 21, 25, 29, 32, 38, 40, 41, 45, 50, 54, 62, 63, 64, 68, 72, 73, 74, 76(II), 77; Crepis biennis L. [Ken T 16] 14; Crepis capillaris (L.) Wallr. [Sp T 9] 2, 4, 25, 27, 72, 73, 76; Crepis tectorum L. [Ap T 16] 45, 46, 50; Erigeron acris L. [Ap H 5] 32; Erigeron annuus (L.) Pers. [Ken T 13] 3(II), 6(II), 8, 9, 11(III), 12, 13, 14(II), 15, 17, 19, 20, 21, 22, 23, 25(III), 26, 27, 28, 29, 30(II), 32, 33, 36, 38, 40(II), 41, 43, 44, 45(II), 46(III), 49, 51(III), 54, 55(II), 57, 58, 62(II), 63, 64(II), 68(II), 69, 71, 72, 73, 74(II), 76, 77; Erigeron ramosus (Walters) Britton, Sterns \& Poggenb. [Ken T 13] 3, 4, 10(III), 25, 29, 30, 31, 33, 51; Eupatorium cannabinum L. [Sp H 7] 25, 26, 75; Filago minima (Sm.) Pers. [Ap T 5] 28, 77(II); Gaillardia pulchella Foug. [D T 19] 73; Galinsoga ciliata (Raf.) S. F. Blake [Ken T 16] 3, 4, 6, 10, 29, 32, 33, 35, 51, 54, 58, 64, 72, 73, 74, 76; Galinsoga parviflora Cav. [Ken T 16] 3, 9,
$10,11,17,21,24,25,27,29,30,31,32,33,38,40$ (II), 45, 49, 50, 51, 54(II), 55, 58, 62, 64, 68, 71, 72, 73, 77; Gnaphalium sylvaticum L. [Ap H 2] 20, 22, 28, 29, 42, 71; Gnaphalium uliginosum L. [Ap T 12] 72; Helianthus tuberosus L. [Ken G 13] 22, 45, 64; Helichrysum arenarium (L.) Moench [Ap H 5] 22, 55, 77; Heliopsis scabra Dunal [D H 19] 9; Hieracium lachenalii C. C. Gmel. [Sp H 2] 19; Hieracium murorum L. [Sp H 2] 44, 61, 65; Hieracium pilosella L. [Ap H 5] 4, 5, 12, 13, 17, 19(II), 21, 22, 25, 28, 30, 31, 32(II), 38, 41, 42, 43(II), 44, 45, 46, 47, 50, 53, 55(II), 61, 62, 65, 68, 69, 71, 72, 73, 74(II), 76, 77, 78; Hieracium sabaudum L. [Sp H 2] 1; Hypochoeris radicata L. [Ap H 5] 4, 11, $12,13,17,21,25,28,44,45,47,50,65,71,72,73,74$, 77; Inula britannica L. [Ap H 10] 9; Lactuca serriola L. [Arch T 15] 14, 27, 28, 33, 35, 38(III), 46, 51, 54; Lapsana communis L. s.s. [Ap T 3] 14, 18, 27, 35, 47, 48, 51, 58; Leontodon autumnalis L. [Ap H 10] 3, 4, 10, 21, 22, 25, 32(II), 33, 40, 41, 45, 46, 47, 51, 53, 54, 58, 63, 64, 67, 68, 71, 72, 73(II); Leucanthemum vulgare Lam. s.s. [Ap H 9] 5, 9, 13, 25, 30, 35, 38, 50, 72; Mycelis muralis (L.) Dumort. [Sp H 1] 2, 4, 15, 16, 18 , 23, 24, 25, 27, 28, 48, 49, 60, 66; Picris hieracioides L. [Ap H 14] 14, 28, 33, 38, 76; Rudbeckia hirta L. [Ken T 19] 2, 3(II), 4, 6, 9, 10, 11, 13, 14, 16, 17, 21, 25, 31, 32, 40, 41, 45, 46, 50, 51, 54, 55, 62, 63, 64, 68(II), 72, 73(II), 74, 76, 77; Rudbeckia laciniata L. [Ken G 7] 2, 3, 9, 10, 14, 17, 22, 29, 30, 31, 38, 40, 46, 51, 54, 64, 73; Senecio jacobaea L. [Ap H 4] 3, 25, 45; Senecio sylvaticus $\mathrm{L}$. [Ap T 2] 42; Senecio viscosus $\mathrm{L}$. [Ap T 15] 13, 14, 16, 24, 43, 50, 67; Senecio vulgaris L. [Arch T 16] $6,11,25,40,51,53,54,55,58,62,63,64,72,73$; Solidago canadensis L. [Ken G 13] 11, 14, 25, 50, 51, 54(II), 64(III), 74, 76; Solidago gigantea Aiton [Ken G 13] 2, 6, 7 (V), 9, 10, 14, 16, 17, 18, 21(II), 22, 3, 24, 25(II), 27, 29, 30, 31(II), 32(III), 33(II), 43(II), 46, 47(II), 50, 64, 78; Solidago virgaurea L. s.s. [Sp H 2] 19, 20, 24, 25, 28, 31(II), 32, 43, 44, 50, 51, 61, 65, 68, 69, 74, 76, 78; Sonchus arvensis L. [Ap H 16] 2, 3, 4, 9, 11, 14, 35, 40, 41, 50, 51, 54, 58, 63, 64, 72; Sonchus asper (L.) Hill [Arch T 16] 35, 38; Sonchus oleraceus L. [Arch T 16] 6, 9, 10, 14, 25, 32, 33, 35(II), 38, 40(II), 41, 51, 54, 58, 62, 72, 76; Tagetes patula L. [D T 19] 45, 63; Tanacetum parthenium (L.) Sch. Bip. [Ken $\mathrm{H}$ 16] 6, 10, 16, 21, 25; Tanacetum vulgare $L$. [Ap H 13] $2,3,6,8,9,11,15,16,21,22,24,25,28,29,30,31$, $32,33,40,41,45,46,50,51,54,57,62,63,64,71,72$, 73, 74, 77; Taraxacum officinale Webb. [Sp H 9] 1, 3, 4, 5, 8, 9(II), 10(II), 12, 13, 14(II), 17(II), 21, 22, 25, 26, 27, 29, 30(II), 31, 32(II), 33(III), 34, 35, 36, 37, 38, 40(II), 41(III), 44, 45(II), 46(II), 47, 49, 50, 51(II), 53, 54(III), 56, 58(II), 62(III), 63(II), 64(II), 65, 67, 68(II), 69, 71, 72(III), 73(IV), 74(III), 76; Tragopogon dubius Scop. [Ap T 14] 38, 42; Tragopogon pratensis L. s.s. [Ap T 9] 62; 
Liliaceae: Allium oleraceum L. [Ap G 4] 14, $15,22,29,35,38,40,62 ;$ Asparagus officinalis $L$. [Ken G 14] 3, 6, 7, 9, 10, 11, 13, 14, 16, 17, 21, 25, 28, $29,30,32,35,38,40,41,43,45,46,47,51,54,62,63$, 64, 68, 72, 73, 74, 76, 78; Convallaria majalis L. [Sp G 2] 1, 3, 6, 9, 11, 12, 13, 14, 16, 17, 21, 25, 27, 28, $29,31,32,36,37,38,43,44,47,49,51,54,58,62,63$, $64,66,68,71,72,73,74,76 ;$ Hemerocallis sp. [Ken $\mathrm{H}$ 16] $2,9,10,11,12,14,15,16,17,20,21,22,23,24$, $25,27,28,29,30,31,32,34,35,36,37,38,39,41,42$, 43, 44, 47 (II), 49, 51, 54, 57, 59, 62, 63, 64, 67, 68, 69, 72, 73, 75, 76; Lilium bulbiferum L. [Sp G 1] 59, 75; Maianthemum bifolium (L.) F. W. Schmidt [Sp G 2] 20, 24, 42, 49, 52, 65; Polygonatum multiflorum (L.) All. [Sp G 1] 9, 18, 31, 33, 36; Polygonatum odoratum (Mill.) Druce [Sp G 2] 43 (II);

Iridaceae: Iris germanica L. [Ken H 16] 22, $38,57,67,76$; $\underline{\text { ris sibirica }}$ L. [Sp H 8] 3, 5, 11, 17, 25 , $35,50,58,62,64,75,76$;

Juncaceae: Juncus effusus L. [Ap H 2] 42, 60; Luzula pilosa (L.) Willd. [Sp H 2] 20, 23, 44, 66, 70;

Poaceae: Agrostis capillaris L. [Ap H 5] 1, 2, 7, $8,12,13,16,17,19,21,22,23,25,28,30,31,32,42$, $43,44,46,47,50,51,53,55,61,62,63,65,66,67,68$, 69, 72, 74, 78; Agrostis gigantea Roth [Ap G 8] 6, 9, 14, 21, 22, 25, 26, 29 (II), 33, 41, 45, 46, 50, 51, 54, 62, 63, 67, 71, 72, 73, 75, 76, 77; Agrostis vinealis Schreb. [Sp H 5] 43, 44; Anthoxantum odoratum L. s.s. [Ap H 2] 16; Apera spica-venti (L.) P. Beauv. [Arch T 17] 14, 21, 40, 46, 51, 55 (II), 62, 63, 64, 72, 74; Arrhenatherum elatius (L.) P. Beauv. Ex J. Presl \& C. Presl [Ap H 9] 3, 14, 21, 29, 30, 31, 32, 33, 38, 40, 41, 43, 45, 62, 63, 64, 75; Brachypodium sylvaticum (Huds.) P. Beauv. [Sp H 1] 12, 23, 35, 37; Briza media L. [Sp H 9] 53; Bromus hordeaceus L. [Ap T 14] 11, 21, 40, 45, 50, 54, 55, 72, 73, 77; Bromus inermis Leyss. [Ap G 14] 2, 3, 40, 43, 54, 63, 75; Bromus tectorum $\mathrm{L}$. [Arch $\mathrm{T}$ 15] 11, 76; Calamagrostis arundinacea (L.) Roth [Sp H 2] 9; Calamagrostis epigejos (L.) Roth [Ap G 2] $1,2,3,5,6,7,10,11,12,13,14,16,19,20,22$, $25,26,27,28,30,32,33,36,43,44,45,50,51,53$, 54, 55, 62, 63, 64, 68, 71, 72, 73, 74, 75, 76 (II), 77; Corynephorus canescens (L.) P. Beauv. [Ap H 5] 13, 21, 43, 55, 69, 76, 77; Dactylis glomerata L. [Ap H 9] $9,14,15,17,26,28,29,30,32,38,40$ (II), 41, 45, 46, 49, 51, 52, 53, 54, 58, 62, 63 (II), 64, 66, 68, 75; Dactylis polygama Horv. [Sp H 1] 14, 48; Danthonia decumbens DC. [Sp H 2] 44, 65, 78; Deschampsia caespitosa (L.) P. Beauv. [Sp H 8] 18, 20, 23, 26, 27, 41, 47, 48, 53, 60; Deschampsia flexuosa (L.) Trin. [Sp H 5] 24, 42, 44, 66; Digitaria ischaemum (Schreb.) H. L. Mühl.[Arch T 16] 3, 5, 6, 9, 11, 21, 25, 28, 29, 30, 31, $32,41,43,45,50,51,54,55,62,64,68,71,72,73,74$, 76, 77; Digitaria sanguinalis (L.) Scop. [Arch T 15] 11, 13, 14, 45, 54, 62, 77; Echinochloa crus-galli (L.)
P. Beauv. [Arch T 16] 3, 6, 9, 10, 14, 31, 32, 33, 35, 38, 40 (II), 41, 45, 46, 51, 54, 55, 58 (II), 62, 72, 73 (II); Elymus repens (L.) Gould [Ap G 10] 2, 6, 9, 14, 15, 16, 17, 22, 25, 28, 29, 31, 41, 42, 43, 46, 47, 50, 51, 53, 67, 69, 72, 73, 74, 75, 76; Eragrostis albensis [Ken T 16] 11; Festuca gigantea (L.) Vill. [Sp H 1] 12, 14, 15, $24,25,26,27,33,48,49,52,60$; Festuca ovina L. s.s. [Sp H 2] 16, 19, 42, 61, 65, 66, 78; Festuca pratensis Huds. [Ap H 9] 25, 62, 68; Festuca rubra L. s.s. [Ap H 9] 9, 14, 16, 28, 30, 35, 41, 42, 43, 53, 57, 64, 67, 68, 69, 71, 75, 76; Festuca trachyphylla (Hack.) Krajina [Ap H 5] 44; ×Festulolium adscendens (Retz.) Asch. \& Graebn. [Ap H 16] 54; Holcus lanatus L. [Ap H 8] 2, 4, 32, 46, 53, 71, 72, 74; Koeleria glauca (Spreng.) DC. [Ap H 5] 43; Lolium perenne L. [Ap H 10] 10, 11, 17, 22 (II), 25, 26, 27, 29, 30, 31, 33, 35, 38, 40, 41, 45, 47, 51, 54, 58, 62, 63, 64, 68, 72, 73, 74, 76; Melica nutans L. [Sp H 2] 36; Milium effusum L. [Sp H 1] 23, 60; Nardus stricta L. [Sp H 2] 13, 61, 65, 76; Panicum milliaceum L. [D T 19] 6; Phalaris arundinacea $\mathrm{L}$. [Ap H 7] 3, 25, 51; Phalaris arundinacea $\mathrm{L}$. var. picta L. [Ap H 7] 3, 9, 11, 14, 22, 29, 45, 51, 54, 68, 73 (II), 74; Phleum pratense L. [Ap H 9] 7, 21, 40, 50, 51, 53; Phragmites australis (Cav.) Trin. ex Steud. [Sp Hel 7] 54, 64; Poa annua L. [Ap T 10] 3, 4, 6, 9, 10, 11, 14, 17 (II), 22, 25, 27, 31, 35, 38, 40, 41, 45, 51, 54, 58, 62, 64, 68, 72, 73, 74, 77; Poa compressa L. [Ap H 14] 1, 2, 43, 50, 69, 76, 77; Poa nemoralis L. [Sp H 2] 12, 25, 27, 49, 51, 52; Poa palustris L. [Sp H 7] 47, 51, 69; Poa pratensis L. s.s. [Ap H 9] 9, 22, 43, 71, 72, 73, 74, 77; Poa trivialis L. [Ap H 12] 23, 48; Setaria pumila (Poir.) Roem. \&Schult. [Arch T 16] 2, $3,5,6,7,9,11,14,21,22,25,28,29,31,32,33,38$, $41,45,46,50,51,54,55,58,59,62,63,64,68,72,73$ (II), 74, 76, 77; Setaria viridis (L.) P. Beauv. [Arch T 16] $5,6,11,13,14,17,21,25,29,30,31,32,33,38$, $41,42,43,44,45,46,50,51,54,55,62,63,64,69,72$, 73, 74, 76 (II), 77; Trisetum flavescens (L.) P. Beauv. [Sp H 9] 14, 45, 49;

Cyperaceae: Carex brizoides L. [Sp H 1] 12; Carex caryophyllea Latourr. [Sp H 4] 78; Carex ericetorum Pollich [Ap H 2] 44, 50, 61, 65; Carex hirta L. [Ap G 10] 7, 8, 11, 15, 19, 21, 22, 28, 47, 53, 54, 68, 69, 72 73, 76; Carex ligerica J. Gay [Sp G 1] 16; Carex ovalis Gooden [Ap H 2] 53; Carex pallescens L. [Sp H 2] 16; Carex spicata Huds. [Ap H 2] 12, 13, 18, 22, 23, 26, 33, 35, 36, 40, 43, 52, 71.

\section{Characteristics of flora and discussion}

In the cemeteries of the analysed area, the occurrence of 523 species of vascular plants belonging to 75 families was recorded. The largest number of species belongs to the family Asteraceae (76), followed by those belonging to the families Poaceae (50) and Rosaceae 
(40), as well as Fabaceae (25), Caryophyllaceae (22), and Lamiaceae (20). Six of the above mentioned families account for $45 \%$ of the flora in the analysed cemeteries. Similarly, C e $1 \mathrm{k}$ a and Ż y w i c a (2005), when investigating 7 cemeteries in the Wielkopolska region, showed that the families Asteraceae and Poaceae are the most abundant in the number of species, followed by Caryophyllaceae. A considerable proportion of species from the family Poaceae in plant communities of the cemeteries in the city of Lublin was also indicated by Mosek and Miazga (2005). They are solely native, uncultivated species. They develop in abundance in unshaded or moderately shaded locations.

In the cemeteries of the Roztocze region, a total of 9 fern species were recorded. Matteucia struthiopteris is cultivated in cemeteries, but it tends to spread vegetatively. Among ferns, there are species found only in cracks of walls surrounding cemeteries and in cracks of gravestones (Asplenium ruta-muraria, A. trichomanes, Cystopteris fragilis). These species, due to the presence of stone walls, are frequent components of floras in urban cemeteries ( $\mathrm{M} \mathrm{a} \mathrm{c} \mathrm{i} \mathrm{e} \mathrm{j} \mathrm{c} \mathrm{zak} \mathrm{2008).}$

The frequency scale for species is extensive and ranges from 1 to 61 localities. "Very rare species" (present at 1-2 localities) predominate, comprising 194 taxa, which accounts for $37 \%$ of the flora (Fig. 2). The category of "common species" (occurring at 51-78 localities) consists of plants of the herbaceous layer, i.e. Aegopodium podagraria, Conyza canadensis, Taraxacum officinale, Vinca minor, Galeopsis pubescens and Urtica dioica, as well as two phanerophytes - Tilia cordata and Robinia pseudoacacia - forming the tree layer and regenerating in abundant numbers in the herbaceous layer. The group of common species is characterised by a higher mean value of cemetery area cover in comparison with the species representing the other frequency classes (Fig. 2).

In the analysed cemeteries, the richest layer was the herbaceous layer comprising 481 species, which accounts for $92 \%$ of recorded vascular plants. In the shrub layer, a total of $74(14 \%)$ species were recorded, while the tree layer was formed by 34 species $(6 \%)$. The plant division presented above corresponds to the percentage proportions of life forms (Fig. 3). The percentages of mega- and nanophanerophytes are very similar, with both groups comprising a total of $15 \%$ of the flora. Similar proportions of trees and shrubs were observed in the suburban cemeteries of the city of Warsaw (Galer a et al. 1993). A study by S u dnik-Wójcikowska (1987) confirmed that in urbanised areas cemeteries (next to city parks) are important localities of the occurrence of phanerophytes. Native and alien trees and shrubs comprise an important element in the spatial composition of contemporary necropolises.
Among plants with non-woody shoots, hemicryptophytes (45\%) and therophytes (32\% of the flora) predominate. The presence of therophytes is distinctly marked in the presently used cemeteries, where tending measures are regularly applied. Hemicryptophytes are connected with both presently used and closed cemeteries. The dominance of hemicryptophytes and therophytes is typical of not only cemeteries $(\mathrm{C} \mathrm{e} 1 \mathrm{k} \mathrm{a}$ and Zywica, 2005; Czarna et al. 2007), but of urban floras in general (S u d n i k - W ó j c i k o w s ka, 1987; J a c k ow i a k, 1990).

Native species comprise almost $67 \%$ of the flora of the cemeteries, while the other $33 \%$ are composed of anthropophytes (Fig. 4). Such a distribution of proportions for native and alien species is typical of most necropolises (C e l k a and $\dot{Z}$ y wi c a, 2004; C z ar $\mathrm{n} a, 2004)$. In Jewish cemeteries, where ornamental species are not introduced as a rule, the proportion of anthropophytes is small ( $\mathrm{Czarna}, 2004$; $\mathrm{Cz}$ arna and N o w iń s k a, 2010).

Among permanently naturalised alien species, kenophytes (16\%) predominate over archeophytes $(10 \%)$. A total of 39 species of alien origin do not show signs of permanent naturalisation, thus they were classified as diaphytes. Percentages of species representing 19 socio-ecological groups are presented in Fig. 5. A group of plants connected with weed communities of gardens and root crops, Polygono-Chenopodietalia (group 16), is represented in considerable numbers. The anthropogenic character of the cemeteries is also manifested in a high proportion of plants of unknown phytosociological affiliation (group 19). On the other hand, a still considerable and similar proportion was also recorded for these native species which are connected with fertile broad-leaved forests and shrub communities, i.e. Fagetalia, Prunetalia (group 1), as well as acidophilous or xerothermic oak forests, mixed coniferous forests and their substitute shrub, herb or grassland communities, i.e. Quercion robori-petraeae, Epilobion and Nardetalia (group 2).

Over $25 \%$ of vascular plants recorded in the analysed cemeteries are cultivated plants. In this group, geographically alien species predominate over native plants (99 vs. 43 species). According to Czarna (2001), cemeteries are testing grounds where previously cultivated species have a chance to survive. Such constant cemetery plants in the analysed cemeteries include 41 species: Amaranthus caudatus, A. cruentus, Aquilegia vulgaris, Asparagus officinalis, Aster novi-belgii, Calendula officinalis, Convallaria majalis, Cosmos bipinnatus, Dianthus barbatus, Erigeron annuus, E. ramosus, Euphorbia marginata, Hemerocallis sp., Hesperis matronalis, Iris germanica, Kochia scoparia, Lamium album, Leonurus cardiaca, Leucanthemum vulgare, Lupinus polyphyllus, Matteucia struthiopteris, Oxalis corniculata, Phalaris arun- 
dinacea var. picta, Polygonatum multiflorum, Reynoutria japonica, Rosa xfrancofourtana, Rosa majalis, $R$. rugosa, Rudbeckia hirta, $R$. laciniata, Salvia verticillata, Saponaria officinalis, Sedum spurium, Solidago canadensis, S. gigantea, Sorbaria sorbifolia, Spiraea chamedrifolia Symphoricarpos albus, Syringa vulgaris, Vinca minor and Viola odorata.

Some interesting and valuable species can be found in the investigated cemeteries. Carex ligerica is a new species for the investigated region. A closed cemetery in Gorajec (Cieszanów commune) is the farthest east area occupied by this species in Poland. Geranium sibiricum found in Tomaszów Lubelski is considered to be a very rare plant in Poland ( $\mathrm{Z}$ a j a c , $\mathrm{Z}$ a $\mathrm{j}$ ą c 2001). The necropolises of the investigated region are located in different habitats and differ in respect to anthropopression intensity. For this reason, they are notable as the mainstay of forest plants not very frequent in Poland (e.g. Carex brizoides, Euonymus verrucosa, Lembotropis nigricans, Vicia dumetrum, Viola collina), plants of forest edges (Astragalu cicer, Geranium phaeum, Salvia verticillata) and meadows (Cirsium rivulare), or ruderal plants (Elsholtzia ciliata and Fumaria vaillantii).

In the course of the study on the presently used cemetery in Ciechanów, Eragrostis albensis was found. It is a species described for the first time in science on the basis of herbarium specimens coming from the area of the middle Elbe River (S c h o l z, 1996). Previously, plants from the genus Eragrostis found on the Elbe were considered to be E. pilosa. In south-eastern Poland, the expansion of this species is observed in anthropogenic habitats ( $\mathrm{Michalewska}$ and $\mathrm{No}$ b i s z, 2005).

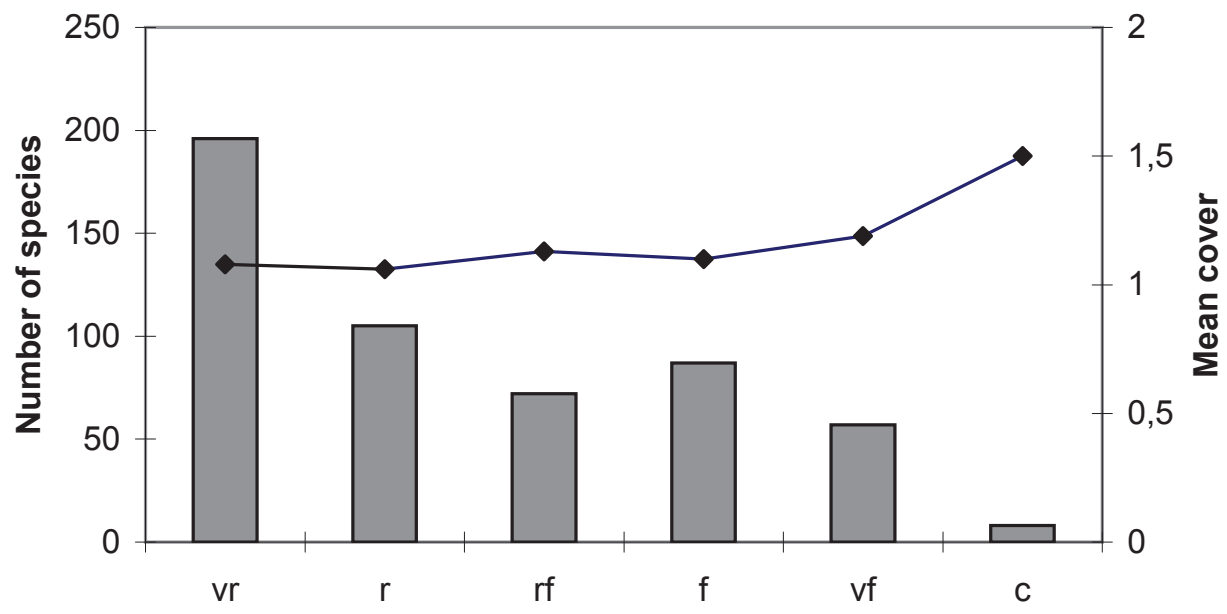

Fig. 2. The number of species and their average cover in particular frequency classes in the investigated cemeteries of the Roztocze region.

Explanations: vr - very rare; $\mathrm{r}$ - rare; $\mathrm{rf}$ - relatively frequent; $\mathrm{f}$ - frequent; vf - very frequent; $\mathrm{c}$ - common. Columns present the number of species, the line presents mean cover of species.

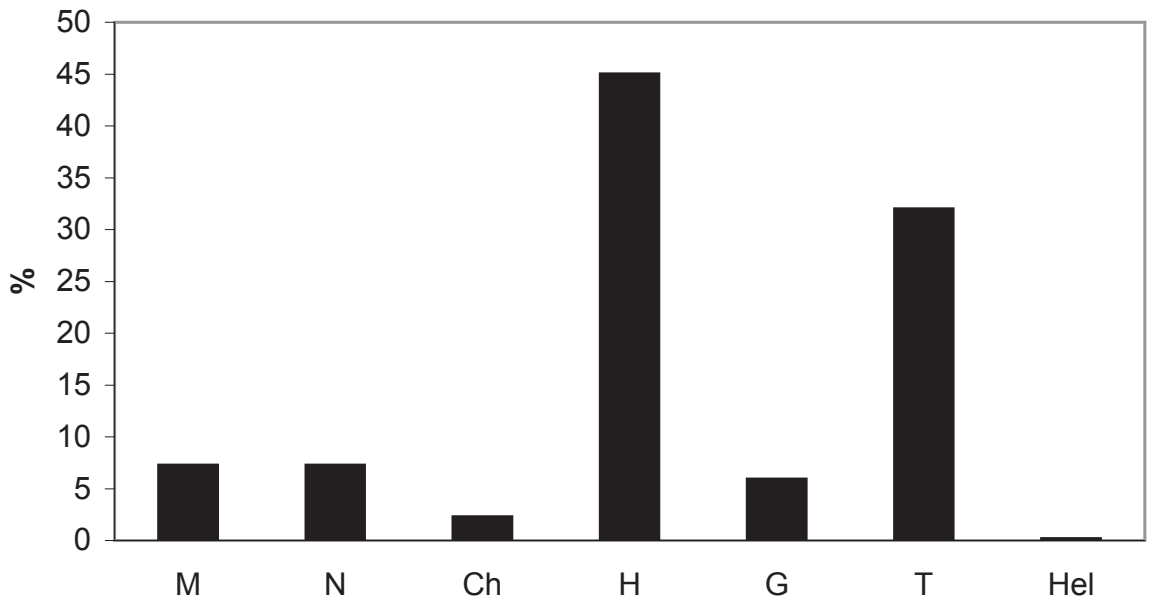

Fig. 3. The participation of life forms in the flora of the investigated cemeteries in the Roztocze region.

Explanations: $\mathrm{M}$ - megaphanerophytes; $\mathrm{N}$ - nanophanerophytes; $\mathrm{Ch}$ - chamaephytes; $\mathrm{H}$-hemicryptophytes; $\mathrm{G}$ - geophytes; $\mathrm{T}$ - therophytes; Hel - helophytes. 


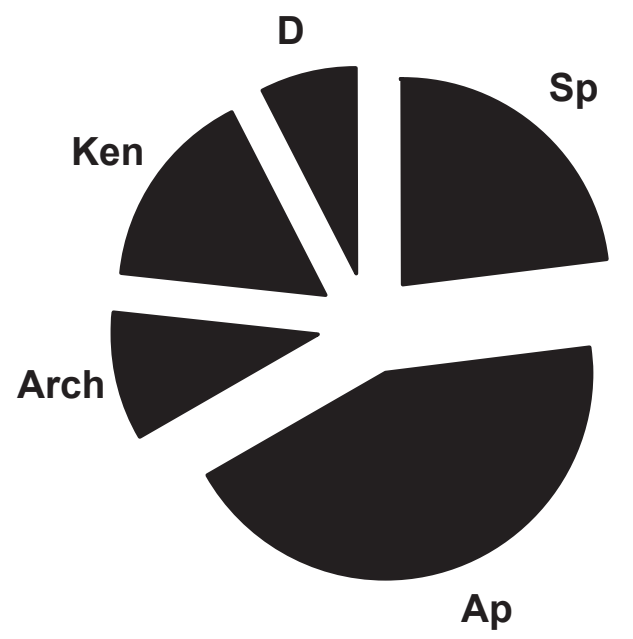

Fig. 4. Spectrum of geographical-historical groups in the flora of the cemeteries in the Roztocze region.

Explanations: Sp - spontaneophytes; Ap - apophytes; Arch - archaeophytes; Ken -kenophytes; D - diaphytes.

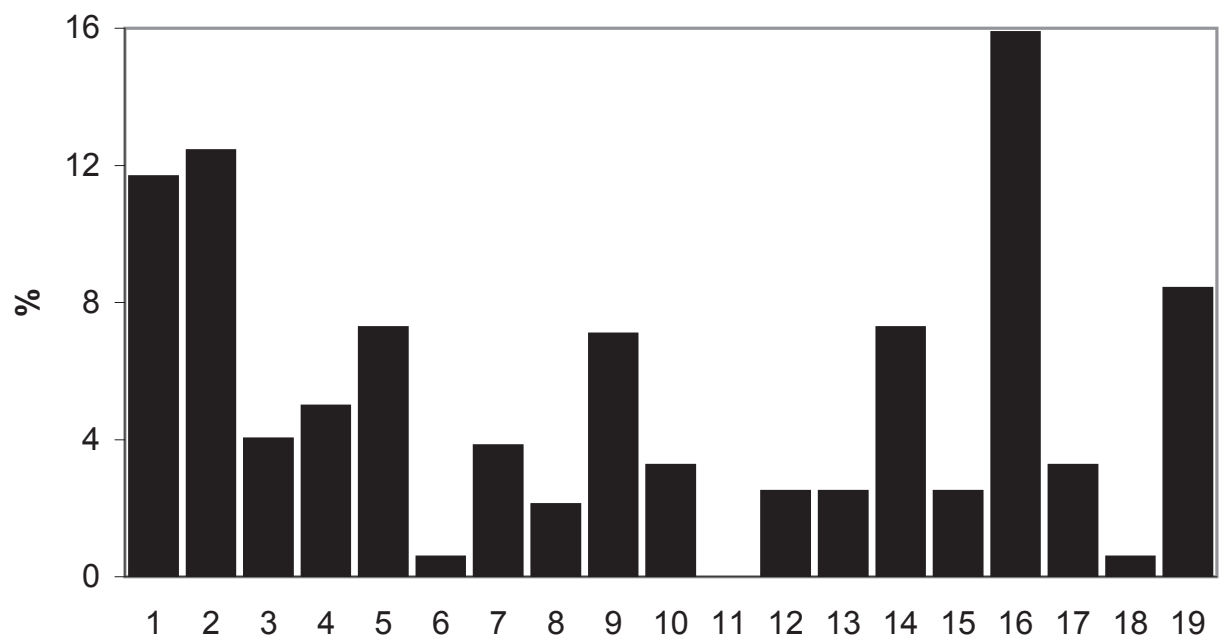

Fig. 5. The participation of socio-ecological groups in the flora of the cemeteries in the Roztocze region.

Explanations - see the "Material and Methods" chapter.

\section{ACKNOWLEDGEMENTS}

Collected trees and shrubs were identified by Professor Jerzy Zieliński from Kórnik, to whom we would like to express our sincere thanks.

\section{REFERENCES}

Brandes S., Brandes D., 1996. Flora und Vegetation von Dörfern im westlichen Sachsen-Anhalt. Braunschw. Naturkdl. Sehr. 5 (1): 165-192.

Celka Z., Żywica J., 2004. Flora naczyniowa wybranych cmentarzy Ostrowa Wielkopolskiego i okolicy. I Vascular flora of chosen cemeteries of Ostrów Wielkopolski and its surroundings (Wielkopolska). Rocz. AR Pozn. 363, Bot. 7: 11-31. (in Polish)

Chmiel J., 1993. Flora roślin naczyniowych wschodniej części Pojezierza Gnieźnieńskiego i jej antropogenicz- ne przeobrażenia w wieku XIX i XX. Część II. Atlas rozmieszczenia roślin. ss. 212. Wydawnictwo Sorus, Poznań. (in Polish)

Czarna A., 2001. Flora naczyniowa cmentarzy ewangelickich w Koźminie i Koźmińcu (Nizina Wielkopolska). I Vascular flora of evangelical cemeteries in Koźmin and Koźminiec (Wielkopolska Lowland). Rocz. AR Pozn. 334, Bot. 4: 27-37. (in Polish)

Czarna A., 2004. Flora naczyniowa cmentarzy na terenie Jarocina. / Vascular flora of cemeteries in the town of Jarocin. Rocz. AR Pozn. 363, Bot. 7: 33-45. (in Polish)

Czarna A., Nowińska. R., Wysakowska I., 2007. Vascular flora of the municipal cemetery in Ustrzyki Dolne (Bieszczady Mts., Poland). Rocz. AR Pozn. 386, Bot.-Stec. 11: 29-33.

Czarna A., Nowińska R., 2010. Vascular plants of certain old Jewish cemeteries in Western Carpathians. Rocz. AR Pozn. 389, Bot.-Stec. 14:45-52. 
Fijałkow ski D., 1994. Flora roślin naczyniowych Lubelszczyzny. Tom 1. Lubelskie Towarzystwo Naukowe. (in Polish)

Galera H., Sudnik-Wójcikowska B., Lisowsk a M., 1993. Flora cmentarzy lewobrzeżnej Warszawy na tle flory miasta. / Flora of the cemeteries of the left-bank part of Warsaw compared to the flora of the whole city. Fragm. Florist. Geobot. 38 (1): 237-261. (in Polish)

J a c k ow i a k B ., 1990 Antropogeniczne przemiany flory roślin naczyniowych Poznania. Ser. Biol. 42. Wyd. Nauk. UAM, Poznań. (in Polish)

Kolbuszewski J., 1996. Cmentarze. Wydawnictwo Dolnośląskie, Wrocław. (in Polish)

Maciejczak B., 2008. Resources and distribution of pteridophytes in the area of Kielce (SE Poland). In: E. Szczęśniak, E. Gola (eds), Club mosses, horsetails and ferns in Poland - resources and protection. Polish Botanical Society \& Institute of Plant Biology, University of Wrocław, Wrocław: 117-125.

Michalewska A., Nobisz M., 2005. Ekspansja Eragrostis albensis (Poaceae) na antropogenicznych siedliskach w południowo-wschodniej Polsce. / Expansion of Eragrostis albensis (Poaceae) on anthropogenic sites in the south-eastern Poland. Fragm. Flor. Geobot. Polonica, 12(1): 45-55. (in Polish)

Mirek Z., Piękoś-Mirkowa H., Zając A., Zają c M., 2002., Flowering Plants and Pteridophytes of Poland a Checklist. Biodiversity of Poland, 1. W. Szafer Institute of Botany, Polish Academy of Science, Kraków.

Mosek B., S. Miazga S., 2005. Grasses in plant communities of cemeteries in Lublin (research note). Łąkarstwo w Polsce, 8: 289-296.

Rut kowski L., 1998. Klucz do oznaczania roślin naczyniowych Polski niżowej. Wydawnictwo Naukowe PWN, Warszawa. (in Polish)

Scholz H., 1996. Eragrostis albensis (Gramineae), des Elb-Leibesgras - ein neuer Neo-Endemit Mitteleuropas. - Verh. Bot. Ver. Berlin Brandenburg, 128: 73-82 (in German).

Sudnik-Wójcikowska B ., 1987. Flora miasta Warszawy i jej przemiany w ciągu XIX i XX wieku. Część I. / Flora of the city of Warsaw and changes in the 19th and 20th centuries. Part I. Wydawnictwo Uniwerstetu Warszawskiego, Warszawa. (in Polish)
W ład P., Wiśniewski M., 2004. Roztocze Wschodnie. Wydawnictwo Naukowe, Turystyczne i Edukacyjne, Mielec. (in Polish)

Zarzycki K., Trzcińska-Tacik H., Różański W., Szeląg Z., Wołek J., Korzennik U., 2002. Ecological Indicator Values of Vascular Plants of Poland. W. Szafer Institute of Botany, Polish Academy of Science, Kraków.

\section{Flora naczyniowa cmentarzy Roztocza i terenów przyległych}

\section{Streszczenie}

Praca przedstawia wyniki badań florystycznych 78 cmentarzy Roztocza oraz granicznych terenów należących do sąsiednich mezoregionów: Płaskowyżu Tarnogrodzkiego i Równiny Bełskiej. Flora badanych nekropolii jest bogata, składają się na nią 523 gatunki roślin naczyniowych. 92\% gatunków występuje w warstwie zielnej. Są to głównie hemikryptofity $(45 \%)$ i terofity (32\% flory), przy czym obecność terofitów zaznacza się wyraźniej na cmentarzach czynnych, gdzie prowadzone są systematyczne zabiegi pielęgnacyjne. Antropogeniczny charakter nekropolii wyraża się zwiększonym udziałem gatunków roślin związanych ze zbiorowiskami chwastów ogrodowych i polnych upraw okopowych (Polygono-Chenopodietalia) oraz roślin o nieokreślonej bliżej przynależności fitosocjologicznej.

Ponad 25\% gatunków stwierdzonych na badanych cmentarzach to rośliny uprawiane. 41 taksonów zostało posadzonych na terenie nekropolii, a po zaniechaniu pielęgnacji rosną $\mathrm{w}$ dobrej kondycji do dnia dzisiejszego, czy wręcz rozprzestrzeniają się. Rośliny te należą do dobrych fitoindykatorów miejsc cmentarnych. Najczęściej występującymi reprezentantami tej grupy są: Asparagus officinalis, Convallaria majalis, Cosmos bipinnatus, Erigeron annuus, Euphorbia marginata, Hedera helix, Kochia scoparia, Lupinus polyphyllus, Rudbeckia hirta, Saponaria officinalis, Solidago gigantea, Syringa vulgaris, Symphoricarpos albus, Vinca minor $i$ Viola odorata. 